\title{
LINEAR, FIRST AND SECOND ORDER, UNCONDITIONALLY ENERGY STABLE NUMERICAL SCHEMES FOR THE PHASE FIELD MODEL OF HOMOPOLYMER BLENDS
}

\author{
XIAOFENG YANG*
}

\begin{abstract}
In this paper, we develop a series of efficient numerical schemes to solve the phase field model for homopolymer blends. The governing system is derived from the energetic variational approach of a total free energy, that consists of a nonlinear logarithmic Flory-Huggins potential, and a gradient entropy with a concentrationdependent de-Gennes type coefficient. The main challenging issue to solve this kind of models numerically is about the time marching problem, i.e., how to develop suitable temporal discretizations for the nonlinear terms in order to preserve the energy stability at the discrete level. We solve this issue in this paper, by developing the first and second order temporal approximation schemes based on the "Invariant Energy Quadratization" method, where all nonlinear terms are treated semi-explicitly. Consequently, the resulting numerical schemes lead to a symmetric positive definite linear system to be solved at each time step. The unconditional energy stabilities are further proved. Various numerical simulations of $2 \mathrm{D}$ and $3 \mathrm{D}$ are presented to demonstrate the stability and the accuracy of the proposed schemes.
\end{abstract}

Key words. Phase-field, Cahn-Hilliard, Linear, Homopolymer blends, Invariant Energy Quadratization, Energy Stability.

AMS subject classifications. 65N12 65M12 65M70

1. Introduction. In this paper, we aim to develop some efficient and effective numerical schemes to solve a phase field model for homopolymer blends (PF-HB) (cf. [1,4,6,9]). The evolution PDE system is resulted from the energetic variation of the action functional of the total free energy in the $H^{-1}$ Sobolev space, i.e., the Cahn-Hilliard $(\mathrm{CH})$ equation. The total free energy consists of a logarithmic Flory-Huggins (F-H) type potential, and a gradient entropy with a concentrationdependent de-Gennes type coefficient [9], that is quite similar to the classical CH system [4], in which the free energy consists of the Ginzburg-Landau double well potential, and the gradient entropy with the constant coefficient. This PF-HB model is also referred as the Cahn-Hilliard-Cook model, or the Stochastic-Cahn-Hilliard equation when the PDE system is equipped with an imposed external thermal noise (stochastic term, or called as Cook-noise [6]).

It is well known that the $\mathrm{CH}$ system is one of typical systems of the phase field model with a long history dated back to one century ago (cf. the classical literatures of Rayleigh [30] and Van der Waals [33]). Due to the energetic variational approach applied in the modeling procedure, the exact solutions to the phase field model lead to a decreasing-in-time energy functional. A significant goal in the numerical simulation of the model is the development of algorithms that can verify this property at the discrete level irrespectively of the coarseness of the discretization (in what follows, algorithms of this type will be called unconditionally energy stable or thermodynamically consistent). The unconditionally energy stable scheme is not only critical to capture the correct long time dynamics of the coarse-graining (macroscopic) process, but also can provide flexibility for dealing with stiffness issues. We also remark that the "unconditional" here means the schemes have no constraints for the time step, however, large time step size will definitely induce large errors in practice.

The main challenging issue to solve a variety of phase field models numerically is about the time marching problem, i.e., to design suitable approaches to discretize the nonlinear terms while preserving the energy stability. For the classical $\mathrm{CH}$ equation, the only nonlinear term is the cubic

*Department of Mathematics, University of South Carolina, Columbia, SC 29208, USA. Email: xfyang@math.sc.edu. This author's research is partially supported by the U.S. National Science Foundation under grant numbers DMS-1200487 and DMS-1418898, and the U.S. Air Force Office of Scientific Research under grant number FA9550-12-1-0178. 
polynomial term that is induced from the double well potential. The popular numerical techniques to discretize such terms mainly include the nonlinear convex splitting approach and the linear stabilization approach (cf. $[13,15,20,25,28,31,38]$ and the references therein). However, the PF-HB model is far more complicated than the classical $\mathrm{CH}$ equation since the system has very special nonlinear complexities, where one is the gradient entropy that is accompanied with a non-constant coefficient, and the other is the logarithmic type bulk potential. To the best of the author's knowledge, there does not exist any numerical scheme with provable unconditional energy stabilities for the PF-HB model so far. Moreover, we emphasize that the two prevalent methods mentioned above are actually not applicable for the PF-HB model (cf. the detailed discussions in section 3).

Therefore, in this paper, in order to develop efficient, unconditionally energy stable numerical schemes to solve the PF-HB model, instead of designing special linear stabilizers or constructing convex-concave combinations for nonlinear functionals, we adopt the so-called Invariant Energy Quadratization (IEQ) method, which is a novel approach and had been successfully applied for the polynomial type potentials for other phase field models in the author's work (cf. [19,35, 37]). The essential idea of the IEQ method is to transform the free energy into a quadratic form (since the nonlinear potential is usually bounded from below) of a set of new variables via a change of variables. The new, equivalent system still retains the similar energy dissipation law in terms of the new variables. For the time-continuous case, the energy law of the new reformulated system is equivalent to the energy law of the original system. One great advantage of such a reformulation is that all nonlinear terms can be treated semi-explicitly accordingly, which in turn produces a linear system. Moreover, the resulted linear operator of the system is symmetric positive definite, thus it can be solved by many available efficient linear solovers, e.g., CG, GMRES, or other Krylov subspace methods.

Based on this new approach, we develop some efficient schemes which are accurate (ready for second or even higher order in time), easy-to-implement (linear), and unconditionally energy stable (with a discrete energy dissipation law) to solve the PF-HB model numerically. We rigorously prove the unconditionally energy stabilities for proposed schemes, including the first order backward Euler, the second order Adam-Bashforth, the second order Crank-Nicolson schemes. Through various classical benchmark 2D and 3D simulations, we demonstrate the stability and the accuracy of the proposed schemes as well. To the best of the author's knowledge, the proposed schemes are the first linear and unconditionally energy stable schemes for the PF-HB model.

The rest of the paper is organized as follows. In Section 2, we present the whole model and give the PDE energy law. In Section 3, we develop the numerical schemes and prove the well-posedness of the linear system, as well as the unconditional energy stabilities. In Section 4, we present various 2D and 3D numerical simulations to validate our numerical schemes. Finally, some concluding remarks are presented in Section 5.

2. Model Equations. We now give a brief introduction to the PF-HB model that considers a symmetric homopolymer blend consisting of molecules of type I and II. The average volume fraction of the I-component in the system is $\phi_{0}$. The state of the system is described by the local volume fraction of the component I, $\phi(\boldsymbol{x}, t)$, at all points $\boldsymbol{x} \in \Omega^{d}, d=2,3$ and at time $t$. The phenomenological mesoscopic dynamic equation that relates a temporal change of $\phi(x, t)$ to a local current of the I component, $J_{I}(\boldsymbol{x})$, is governed by the following PF-HB equation (cf. $\left.[1,4,9]\right)$ :

$$
\phi_{t}=-\nabla \cdot J_{I}(\boldsymbol{x}) .
$$

The transport in the system (2.1) follows the difference of the chemical potential. Here, we assume a linear relation between the local current and the gradient of the local chemical potential difference 
$\rho(\boldsymbol{x}, t)[2]$. Thus, the flux $J_{I}$ is given by

$$
J_{I}(\boldsymbol{x}, t)=-\lambda \nabla \rho(\boldsymbol{x}, t),
$$

where $\lambda$ is the Onsager mobility coefficient, and the local chemical potential difference $\rho(x, t)$ is the functional derivative of a coarse-grained free energy functional $\mathbb{E}(\phi)$, i.e.,

$$
\rho(x, t)=\frac{\delta \mathbb{E}(\phi)}{\delta \phi},
$$

with

$$
\mathbb{E}(\phi)=\int_{\Omega}\left(\frac{\nu \alpha^{2}}{36} \kappa(\phi)|\nabla \phi|^{2}+\mathbb{F}(\phi)\right) d \boldsymbol{x}
$$

where,

$$
\kappa(\phi)=\frac{1}{\phi(1-\phi)}
$$

is the gradient energy coefficient of de-Gennes type, $\alpha$ is the Kuhn segment length (cf. [8]). $\mathbb{F}(\phi)$ is the bulk free energy describing the mixture, which is assumed to have the following F-H form,

$$
\mathbb{F}(\phi)=\frac{1}{\nu N}(\phi \ln (\phi)+(1-\phi) \ln (1-\phi))+\chi \phi(1-\phi),
$$

where $\nu$ is the volume of one repeat unit (monomer), $N$ is the degree of the polymerization of the chains, and $\chi$ is the F-H type interaction parameter.

Let $M=\frac{\Lambda}{\nu N}, \epsilon^{2}=\frac{\nu^{2} \alpha^{2} N}{36}, \beta=\chi \nu N$, the final scaled equation of the PF-HB model to be solved numerically has the following form:

$$
\begin{aligned}
& \phi_{t}=M \Delta \mu, \\
& \mu=-2 \epsilon^{2} \nabla \cdot(\kappa(\phi) \nabla \phi)+\epsilon^{2} \kappa^{\prime}(\phi)|\nabla \phi|^{2}+f(\phi),
\end{aligned}
$$

with

$$
\left\{\begin{array}{l}
\kappa^{\prime}(\phi)=\frac{(2 \phi-1)}{\phi^{2}(1-\phi)^{2}}, \\
F(\phi)=\phi \ln (\phi)+(1-\phi) \ln (1-\phi)+\beta\left(\phi-\phi^{2}\right), \\
f(\phi)=F^{\prime}(\phi)=\ln \left(\frac{\phi}{1-\phi}\right)+\beta(1-2 \phi) .
\end{array}\right.
$$

The boundary conditions can be the following two type,

$$
\text { (i) } \phi, \mu \text { are periodic; or (ii) }\left.\partial_{\mathbf{n}} \phi\right|_{\partial \Omega}=\left.\nabla \mu \cdot \mathbf{n}\right|_{\partial \Omega}=0,
$$

where $\mathbf{n}$ is the unit outward normal on the boundary $\partial \Omega$.

When the equation (2.7) is equipped with the external thermal noise $\eta(\boldsymbol{x}, t)$, it becomes the well-known Cahn-Hilliard-Cook model (cf. [1,6,9]), or the Stochastic-Cahn-Hilliard equation, i.e.,

$$
\phi_{t}=M \Delta \mu+\tilde{\eta} \eta(\boldsymbol{x}, t),
$$


where the stochastic term $\eta(\boldsymbol{x}, t)$ represents thermal noise $[2,6,22]$ and satisfies the following fluctuation dissipation relation [2],

$$
E\left[\eta(\boldsymbol{x}, t) \eta\left(\boldsymbol{x}^{\prime}, t^{\prime}\right)\right]=-2 k_{B} T \lambda \Delta \delta\left(\boldsymbol{x}-\boldsymbol{x}^{\prime}\right) \delta\left(t-t^{\prime}\right),
$$

where $\tilde{\eta}>0$ is the scale of magnitude of the noise.

The thermal noise $\eta(\boldsymbol{x}, t)$ can be viewed as an imposed external force. Note that the system (2.11)-(2.8) does not follow the energy dissipation law if the external thermal noise takes into account. Since the main numerical challenge to solve the PF-HB model is to find suitable ways to discretize the nonlinear terms in the chemical potential $\mu$ of (2.8), it is proper to develop numerical schemes and prove their energy stabilities without considering the thermal noise term.

We can easily derive the dissipative energy law for the system (2.7)-(2.8) when the external noise term vanishes $(\tilde{\eta}=0)$. More precisely, by taking the $L^{2}$ inner product of $(2.7)$ with $-\mu$, and of (2.8) with $\phi_{t}$, and performing integration by parts, we obtain

$$
-\left(\phi_{t}, \mu\right)=M\|\nabla \mu\|^{2}
$$

and

$$
\begin{aligned}
\left(\mu, \phi_{t}\right) & =2 \epsilon^{2}\left(\kappa(\phi) \nabla \phi, \nabla \phi_{t}\right)+\epsilon^{2}\left(\kappa^{\prime}(\phi)|\nabla \phi|^{2}, \phi_{t}\right)+\left(f(\phi), \phi_{t}\right) \\
& =\epsilon^{2}\left(\kappa(\phi), \partial_{t}|\nabla \phi|^{2}\right)+\epsilon^{2}\left(|\nabla \phi|^{2}, \partial_{t} \kappa(\phi)\right)+\left(f(\phi), \phi_{t}\right) \\
& =\frac{d}{d t}\left(\epsilon^{2} \kappa(\phi),|\nabla \phi|^{2}\right)+\frac{d}{d t}(F(\phi), 1) .
\end{aligned}
$$

Taking summation of the two equalities, we obtain the energy dissipative law as

$$
\frac{d}{d t} E(\phi)=-M\|\nabla \mu\|^{2} \leq 0
$$

where

$$
E(\phi)=\int_{\Omega}\left(\epsilon^{2} \kappa(\phi)|\nabla \phi|^{2}+F(\phi)\right) d \boldsymbol{x}
$$

Remark 2.1. To clarify the difference between the classical CH equation and the PF-HB model, We present the classical $\mathrm{CH}$ equation for reference. It reads as follows,

$$
\begin{aligned}
& \phi_{t}=M \Delta \mu, \\
& \mu=-\epsilon^{2} \Delta \phi+g(\phi) .
\end{aligned}
$$

where $g(\phi)=G^{\prime}(\phi) . M$ is the mobility function that can be chosen as a constant, or dependent on the phase variable $\phi$. There are two common choices for the nonlinear potential $G(\phi)$, including $G(\phi)=$ $\left(1-\phi^{2}\right)^{2}$ that is the well known double well potential or $G(\phi)=\phi \ln \phi+(1-\phi) \ln (1-\phi)+\beta\left(\phi-\phi^{2}\right)$ as the PF-HB model (cf. [3, 12]). The energy dissipative law for (2.17)- (2.18) is

$$
\frac{d}{d t} E_{C H}(\phi)=-M\|\nabla \mu\|^{2} \leq 0,
$$


where

$$
E_{C H}(\phi)=\int_{\Omega}\left(\frac{1}{2} \epsilon^{2}|\nabla \phi|^{2}+G(\phi)\right) d \boldsymbol{x} .
$$

Note that the coefficient of the gradient entropy is the constant $\frac{1}{2}$, which is different with the coefficient $\kappa(\phi)$ in th PF-HB model.

3. Numerical Schemes. We now develop a set of semi-discrete numerical schemes to solve the PF-HB system (2.7)-(2.8) and present the rigorous energy stabilities. The main challenging issues are to find suitable ways to discretize the nonlinear terms induced from the energy functionals, particularly, the term with the de-Gennes coefficient $\kappa(\phi)$, and the bulk F-H logarithmic potential .

For the classical CH model (2.17)-(2.18), the main topic about the development of time marching schemes is to find suitable discretizations for the cubic polynomial term $g(\phi)$ (cf. $[21,24,26,27,32])$. So far, there are two prevalent numerical techniques for it while preserving the energy stability. The first technique is the so-called convex splitting approach (cf. [13]), where the convex part of the potential is treated implicitly and the concave part is treated explicitly. The convex splitting approach is energy stable, however, it produces a nonlinear scheme at most cases, thus the implementation is complicated and the computational cost is high. The second technique is the so-called stabilization approach (cf. $[5,31,34]$ ), where the term from the nonlinear potential is simply treated explicitly and some linear stabilizing terms are added to improve the stability. Such a linear scheme is extremely easy to implement, however, one of the drawbacks is that its second order version only preserves the conditional energy stability (cf. [31]), i.e., the time step size is controlled by the interfacial thickness $\epsilon$ with the small magnitude.

For the PF-HB model system (2.7)-(2.8), both of the two methods are not optimal choices to preserve the energy stability. First, the stabilization approach is excluded, since (i) we expect to develop temporal second order schemes with unconditional energy stability; (ii) we cannot obtain the energy stability when applying the stabilized approach even for the first order scheme. Second, we do not have natural convex-concave decompositions for the gradient entropy with the de-Gennes coefficient and the logarithmic F-H potential, thus the convex-splitting method is not applicable as well.

Therefore, instead of employing artificial stabilizers or conducting convex splitting iterations, we adopt the novel, so-called IEQ approach to obtain more effective schemes, which are expected to be efficient (linear system with symmetric positive definite), stable (provable unconditionally energy stability), and accurate (ready for second or even higher order in time). The main idea of the IEQ method is to transform the free energy and the PDE system into equivalent forms in terms of new variables via the change of variables. Thus the nonlinear terms can be treated semi-implicitly.

3.1. Equivalent PDE system and its new Energy dissipation law. For the logarithmic $\mathrm{F}-\mathrm{H}$ potential, the domain of the logarithmic energy is an open interval $(0,1)$, thus the numerical solution must be guaranteed strictly inside the domain, otherwise the calculation will be easily overflow. Following the work in [7], we first regularize it from domain $(0,1)$ to $(-\infty, \infty)$, where the logarithmic functional is replaced by a $C^{2}$ continuous, convex, piecewise function. More precisely, for any $\sigma>0$, the regularized $\mathrm{F}-\mathrm{H}$ potential is given by

$$
\widehat{F}(\phi)=\widehat{F}_{1}(\phi)+\beta\left(\phi-\phi^{2}\right),
$$


where

$$
\widehat{F}_{1}(\phi)= \begin{cases}\phi \ln \phi+\frac{(1-\phi)^{2}}{2 \sigma}+(1-\phi) \ln \sigma-\frac{\sigma}{2}, & \text { if } \quad \phi \geq 1-\sigma, \\ \phi \ln \phi+(1-\phi) \ln (1-\phi), & \text { if } \quad \sigma \leq \phi \leq 1-\sigma, \\ (1-\phi) \ln (1-\phi)+\frac{\phi^{2}}{2 \sigma}+\phi \ln \sigma-\frac{\sigma}{2}, & \text { if } \quad \phi \leq \sigma .\end{cases}
$$

Therefore, the domain for the regularized functional is now $(-\infty, \infty)$ and one does not need to worry about that any small fluctuation near the domain boundary $(0,1)$ can cause the overflow.

For the gradient entropy coefficient $\kappa(\phi)$, we regularize it in the similar way. For any $\sigma>0$, we have

$$
\widehat{\kappa}(\phi)= \begin{cases}\frac{1}{\sigma(1-\sigma)}+\frac{1-2 \sigma}{(1-\sigma)^{2} \sigma^{2}}(\phi-(1-\sigma)), & \text { if } \phi \geq 1-\sigma, \\ \frac{1}{\phi(1-\phi)}, & \text { if } \quad \sigma \leq \phi \leq 1-\sigma \\ \frac{1}{\sigma(1-\sigma)}+\frac{2 \sigma-1}{\sigma^{2}(1-\sigma)^{2}}(\phi-\sigma), & \text { if } \phi \leq \sigma .\end{cases}
$$

Thus $\widehat{\kappa}(\phi)$ is positive and $C^{1}$ continuous. For convenience, we consider the problem formulated with the substitute $\widehat{F}(\phi)$ and $\widehat{\kappa}(\phi)$, but omit the ${ }^{\wedge}$ in the notation.

Although $F(\phi)$ is not always positive in the whole domain, we note that it is bounded from below with a uniform lower bound. Thus we could rewrite the free energy functional $F(\phi)$ to the following form,

$$
F(\phi)=(F(\phi)+B)-B
$$

where $B$ is a constant to ensure $F(\phi)+B$ to be positive, for example, $B=1$. In turn we rewrite the free energy to be

$$
E(\phi)=\int_{\Omega}\left(\epsilon^{2}|X(\phi) \nabla \phi|^{2}+(\sqrt{F(\phi)+B})^{2}-B\right) d \boldsymbol{x} .
$$

where $X(\phi)=\sqrt{\kappa(\phi)}$.

We define two auxiliary functions $\boldsymbol{U}(\phi)$ (vector) and $V(\phi)$ (scalar) as

$$
\left\{\begin{array}{l}
\boldsymbol{U}(\phi)=X(\phi) \nabla \phi \\
V(\phi)=\sqrt{F(\phi)+B} .
\end{array}\right.
$$

Thus the total energy becomes

$$
E(\boldsymbol{U}, V)=\int_{\Omega}\left(\epsilon^{2}|\boldsymbol{U}|^{2}+V^{2}-B\right) d \boldsymbol{x} .
$$

Using these new variables $\boldsymbol{U}, V$, we then have the following equivalent PDE system,

$$
\begin{aligned}
& \phi_{t}=M \Delta \mu, \\
& \mu=-2 \epsilon^{2} \nabla \cdot(X(\phi) \boldsymbol{U})+\epsilon^{2} Y(\phi) \boldsymbol{U} \cdot \nabla \phi+Z(\phi) V .
\end{aligned}
$$


By taking the time derivative for the new variables, we obtain

$$
\begin{aligned}
\boldsymbol{U}_{t} & =X(\phi) \nabla \phi_{t}+\frac{1}{2} Y(\phi) \phi_{t} \nabla \phi, \\
V_{t} & =\frac{1}{2} Z(\phi) \phi_{t},
\end{aligned}
$$

where

$$
Y(\phi)=2 X^{\prime}(\phi)=\frac{\kappa^{\prime}(\phi)}{\sqrt{\kappa(\phi)}}, Z(\phi)=\frac{f(\phi)}{\sqrt{F(\phi)+B}}, f(\phi)=F^{\prime}(\phi) .
$$

The transformed system (3.8)-(3.11) in the new variables form a closed PDE system with the following initial conditions,

$$
\left\{\begin{array}{l}
\phi(t=0)=\phi^{0}, \\
\boldsymbol{U}(t=0)=\boldsymbol{U}^{0}=X\left(\phi^{0}\right) \nabla \phi^{0}, \\
V(t=0)=V^{0}=\sqrt{F\left(\phi^{0}\right)+B}
\end{array}\right.
$$

Since the equations (3.10) and (3.11) for the new variables $\boldsymbol{U}, V$ are only differential equations with time, the boundary conditions of the new system (3.8)-(3.11) are still (2.10).

The transformed system (3.8)-(3.11) also follows an energy dissipative laws in terms of the new variables $\boldsymbol{U}, V$. By taking the $L^{2}$ inner product of (3.8) with $-\mu$, of (3.9) with $\phi_{t}$, of (3.10) with $\boldsymbol{U}$, of (3.11) with $V$, performing integration by parts and summing all equalities up, we can obtain the energy dissipation law of the new system (3.8)-(3.11) as

$$
\frac{d}{d t} E(\boldsymbol{U}, V)=-M\|\nabla \mu\|^{2} \leq 0 .
$$

Remark 3.1. The new transformed system (3.8)-(3.11) is exactly equivalent to the original system (2.7)-(2.8) since (3.6) can be easily obtained by integrating (3.10) and (3.11) with respect to the time. Therefore, the energy law (3.14) for the transformed system is exactly the same as the energy law (2.15) for the original system for the time-continuous case.

We emphasize that we will develop energy stable numerical schemes for time stepping of the new transformed system (3.8)-(3.11). The proposed schemes should follow the new energy dissipation law (3.14) instead of the energy law for the original system (2.15).

We fix some notations here. Let $\delta t>0$ denote the time step size and set $t^{n}=n \delta t$ for $0 \leq n \leq N$ with $T=N \delta$. We also denote by $(f(\boldsymbol{x}), g(\boldsymbol{x}))=\int_{\Omega} f(\boldsymbol{x}) g(\boldsymbol{x}) d \boldsymbol{x}$ the $L^{2}$ inner product of any two functions $f(\boldsymbol{x})$ and $g(\boldsymbol{x})$, and by $\|f\|=\sqrt{(f, f)}$ the $L^{2}$ norm of the function $f(\boldsymbol{x})$.

3.2. First-order Scheme. We now present the first order time stepping scheme to solve the system (3.8)- (3.9)-(3.10)-(3.11), where the time derivative is discretized based on the first order backward Euler method.

The first order scheme reads as follows.

Given $\phi^{0}, \boldsymbol{U}^{0}, V^{0}$, we find $\phi^{n+1}, \mu^{n+1}, \boldsymbol{U}^{n+1}, V^{n+1}$ for $n \geq 0$, such that 


$$
\begin{aligned}
& \frac{\phi^{n+1}-\phi^{n}}{\delta t}=M \Delta \mu^{n+1}, \\
& \mu^{n+1}=-2 \epsilon^{2} \nabla \cdot\left(X^{n} \boldsymbol{U}^{n+1}\right)+\epsilon^{2} Y^{n} \boldsymbol{U}^{n+1} \cdot \nabla \phi^{n}+Z^{n} V^{n+1}, \\
& \boldsymbol{U}^{n+1}-\boldsymbol{U}^{n}=X^{n} \nabla\left(\phi^{n+1}-\phi^{n}\right)+\frac{1}{2} Y^{n}\left(\phi^{n+1}-\phi^{n}\right) \nabla \phi^{n}, \\
& V^{n+1}-V^{n}=\frac{1}{2} Z^{n}\left(\phi^{n+1}-\phi^{n}\right),
\end{aligned}
$$

where $X^{n}=X\left(\phi^{n}\right), Y^{n}=Y\left(\phi^{n}\right), Z^{n}=Z\left(\phi^{n}\right)$.

The impressing part of the above schemes is that all nonlinear coefficient of the new variables $\boldsymbol{U}, V$ are treated explicitly, which can tremendously simply the computations. Moreover, in fact, we can rewrite the equations (3.17) and (3.18) as follows,

$$
\left\{\begin{array}{l}
\boldsymbol{U}^{n+1}=\mathbf{A}_{1}+X^{n} \nabla \phi^{n+1}+\frac{1}{2} Y^{n} \nabla \phi^{n} \phi^{n+1}, \\
V^{n+1}=B_{1}+\frac{1}{2} Z^{n} \phi^{n+1},
\end{array}\right.
$$

where

$$
\left\{\begin{array}{l}
\mathbf{A}_{1}=\boldsymbol{U}^{n}-X^{n} \nabla \phi^{n}-\frac{1}{2} Y^{n} \nabla \phi^{n} \phi^{n} \\
B_{1}=V^{n}-\frac{1}{2} Z^{n} \phi^{n}
\end{array}\right.
$$

Thus, the system (3.15)- (3.18) can be rewritten as

$$
\begin{aligned}
& \frac{\phi^{n+1}-\phi^{n}}{\delta t}-M \Delta \mu^{n+1}=0, \\
& \mu^{n+1}-P_{1}\left(\phi^{n+1}\right)=Q_{1} .
\end{aligned}
$$

where

$$
\left\{\begin{aligned}
P_{1}(\phi)= & -2 \epsilon^{2} \nabla \cdot\left(X^{n} X^{n} \nabla \phi\right)-\epsilon^{2} \nabla \cdot\left(X^{n} Y^{n} \nabla \phi^{n} \phi\right) \\
& +\epsilon^{2} Y^{n} X^{n} \nabla \phi^{n} \nabla \phi+\frac{1}{2} \epsilon^{2} Y^{n} Y^{n} \nabla \phi^{n} \cdot \nabla \phi^{n} \phi+\frac{1}{2} Z^{n} Z^{n} \phi \\
Q_{1} \quad & Z^{n} B_{1}-2 \epsilon^{2} \nabla \cdot\left(X^{n} \mathbf{A}_{1}\right)+\epsilon^{2} Y^{n} \mathbf{A}_{1} \cdot \nabla \phi^{n} .
\end{aligned}\right.
$$

Therefore, we can solve $\phi^{n+1}$ and $\mu^{n+1}$ directly from (3.21) and (3.22). Once we obtain $\phi^{n+1}$, the $\boldsymbol{U}^{n+1}, V^{n+1}$ is automatically given in (3.19). Namely, the new variables $\boldsymbol{U}, V$ do not involve any extra computational costs. Furthermore, we notice

$$
\begin{aligned}
\left(P_{1}(\phi), \psi\right)= & 2 \epsilon^{2}\left(X^{n} X^{n} \nabla \phi, \nabla \psi\right)+\epsilon^{2}\left(X^{n} Y^{n} \nabla \phi^{n} \phi, \nabla \psi\right) \\
& +\epsilon^{2}\left(Y^{n} X^{n} \nabla \phi^{n} \nabla \phi, \psi\right)+\frac{1}{2} \epsilon^{2}\left(Y^{n} Y^{n} \nabla \phi^{n} \cdot \nabla \phi^{n} \phi, \psi\right)+\frac{1}{2}\left(Z^{n} Z^{n} \phi, \psi\right) \\
= & \left(\phi, P_{1}(\psi)\right),
\end{aligned}
$$

if $\psi$ satisfies the boundary condition (2.10). Therefore, the linear operator $P_{1}(\phi)$ is symmetric 
(self-adjoint). Moreover, for any $\phi$ with $\int_{\Omega} \phi d \boldsymbol{x}=0$, we have

$$
\begin{aligned}
\left(P_{1}(\phi), \phi\right)= & 2 \epsilon^{2}\left(X^{n} X^{n} \nabla \phi, \nabla \phi\right)+\epsilon^{2}\left(X^{n} Y^{n} \nabla \phi^{n} \phi, \nabla \phi\right) \\
& +\epsilon^{2}\left(X^{n} Y^{n} \nabla \phi^{n} \nabla \phi, \phi\right)+\frac{1}{2} \epsilon^{2}\left(Y^{n} Y^{n} \nabla \phi^{n} \cdot \nabla \phi^{n} \phi, \phi\right)+\frac{1}{2}\left(Z^{n} Z^{n} \phi, \phi\right) \\
= & \epsilon^{2}\left\|\frac{1}{\sqrt{2}} Y^{n} \nabla \phi^{n} \phi+\sqrt{2} X^{n} \nabla \phi\right\|^{2}+\frac{1}{2}\left\|Z^{n} \phi\right\|^{2} \geq 0,
\end{aligned}
$$

where "=" is valid if and only if $\phi^{n}=\frac{1}{2}$ and $\phi=c$ with $c$ is a constant. From the mean zero condition, we have $c=0$.

THEOREM 3.1. The linear operator of the system (3.21)-(3.22) is symmetric (self-adjoint) and positive definite for the variable $\phi^{n+1}$.

Proof. From (3.21), by taking the $L^{2}$ inner product with 1, we derive

$$
\int_{\Omega} \phi^{n+1} d \boldsymbol{x}=\int_{\Omega} \phi^{n} d \boldsymbol{x}=\cdots=\int_{\Omega} \phi^{0} d \boldsymbol{x} .
$$

Let $\alpha_{0}=\frac{1}{|\Omega|} \int_{\Omega} \phi^{0} d \boldsymbol{x}, \beta_{\mu}=\frac{1}{|\Omega|} \int_{\Omega} \mu^{n+1} d \boldsymbol{x}$, and we define

$$
\widehat{\phi}^{n+1}=\phi^{n+1}-\alpha_{0}, \widehat{\mu}^{n+1}=\mu^{n+1}-\beta_{\mu} .
$$

Thus, from (3.21)-(3.22), $\widehat{\phi}^{n+1}$ and $\widehat{\mu}^{n+1}$ are the solutions for the following equations,

$$
\begin{aligned}
& \frac{\psi}{\delta t}-M \Delta \mu=f^{n}, \\
& \mu+\beta_{\mu}-P_{1}(\psi)=g^{n},
\end{aligned}
$$

with

$$
\int_{\Omega} \psi d \boldsymbol{x}=0, \int_{\Omega} \mu d \boldsymbol{x}=0
$$

We define the inverse laplace operator $v=\Delta^{-1} u$ as follows,

$$
\left\{\begin{array}{l}
\Delta v=u, \\
\int_{\Omega} v d \boldsymbol{x}=0,
\end{array}\right.
$$

with the boundary conditions (2.10). Applying $-\Delta^{-1}$ to (3.28) and using (3.29), we obtain

$$
-\frac{1}{M \delta t} \Delta^{-1} \psi+P_{1}(\psi)-\beta_{\mu}=-\Delta^{-1} f^{n}-g^{n} .
$$

Let us express the above linear system (3.32) as $\mathbb{A} \psi=b$, thus, for any $\psi, \phi$ with $\int_{\Omega} \psi d \boldsymbol{x}=0$ and 
$\int_{\Omega} \phi d \boldsymbol{x}=0$, we can easily derive

$$
\begin{aligned}
(\mathbb{A} \psi, \phi) & =\left(-\frac{1}{M \delta t} \Delta^{-1} \psi+P_{1}(\psi)-\beta_{\mu}, \phi\right) \\
& =\left(\psi,-\frac{1}{M \delta t} \Delta^{-1} \phi+P_{1}(\phi)-\beta_{\mu}\right) \\
& =(\psi, \mathbb{A} \phi) .
\end{aligned}
$$

Thus $\mathbb{A}$ is self adjoint. Meanwhile, we derive

$$
\begin{aligned}
(\mathbb{A} \phi, \phi) & =\left(-\frac{1}{M \delta t} \Delta^{-1} \phi+P_{1}(\phi)-\beta_{\mu}, \phi\right) \\
& =\frac{1}{M \delta t}\|\phi\|_{H^{-1}}^{2}+\left(P_{1}(\phi), \phi\right) \geq 0 .
\end{aligned}
$$

The "=" is valid if only if $\phi=0$ from the fact of (3.25). Thus we conclude the theorem.

The stability result of the proposed first order scheme follows the same lines as in the derivation of the new energy dissipation law (3.14).

THEOREM 3.2. The scheme (3.15)-(3.18) is unconditionally stable, and admits a unique solution that satisfies the following discrete energy dissipation law,

$$
\frac{1}{\delta t}\left(E_{1}\left(\boldsymbol{U}^{n+1}, V^{n+1}\right)-E_{1}\left(\boldsymbol{U}^{n}, V^{n}\right)\right) \leq-M\left\|\nabla \mu^{n+1}\right\|^{2},
$$

where

$$
E_{1}(\boldsymbol{U}, V)=\int_{\Omega}\left(\epsilon^{2}|\boldsymbol{U}|^{2}+|V|^{2}-B\right) d \boldsymbol{x}
$$

Proof. The existence and uniqueness of the solution are from Theorem 3.1.

By taking the $L^{2}$ inner product of (3.15) with $-\delta t \mu^{n+1}$, we obtain

$$
-\left(\phi^{n+1}-\phi^{n}, \mu^{n+1}\right)=\delta t M\left\|\nabla \mu^{n+1}\right\|^{2} .
$$

By taking the $L^{2}$ inner product of (3.16) with $\phi^{n+1}-\phi^{n}$, we obtain

$$
\begin{aligned}
\left(\mu^{n+1}, \phi^{n+1}-\phi^{n}\right)= & 2 \epsilon^{2}\left(\boldsymbol{U}^{n+1} X^{n}, \nabla \phi^{n+1}-\nabla \phi^{n}\right)+\epsilon^{2}\left(Y^{n} \boldsymbol{U}^{n+1} \cdot \nabla \phi^{n}, \phi^{n+1}-\phi^{n}\right) \\
& +\left(Z^{n} V^{n+1}, \phi^{n+1}-\phi^{n}\right) .
\end{aligned}
$$

By taking $L^{2}$ inner product of (3.17) with $-2 \epsilon^{2} \boldsymbol{U}^{n+1}$, and applying the following identity

$$
2(a-b, a)=|a|^{2}-|b|^{2}+|a-b|^{2},
$$

we obtain

$$
\begin{aligned}
-\epsilon^{2}\left(\left\|\boldsymbol{U}^{n+1}\right\|^{2}-\left\|\boldsymbol{U}^{n}\right\|^{2}+\left\|\boldsymbol{U}^{n+1}-\boldsymbol{U}^{n}\right\|^{2}\right)= & -2 \epsilon^{2}\left(X^{n} \nabla\left(\phi^{n+1}-\phi^{n}\right), \boldsymbol{U}^{n+1}\right) \\
& -\epsilon^{2}\left(Y^{n}\left(\phi^{n+1}-\phi^{n}\right) \nabla \phi^{n}, \boldsymbol{U}^{n+1}\right) .
\end{aligned}
$$


By taking $L^{2}$ inner product of (3.18) with $-2 V^{n+1}$, we obtain

$$
-\left(\left\|V^{n+1}\right\|^{2}-\left\|V^{n}\right\|^{2}+\left\|V^{n+1}-V^{n}\right\|^{2}\right)=-\left(Z^{n}\left(\phi^{n+1}-\phi^{n}\right), V^{n+1}\right) .
$$

By combining (3.37)-(3.38)-(3.40)-(3.41), we obtain

$$
\begin{aligned}
\epsilon^{2}\left(\left\|\boldsymbol{U}^{n+1}\right\|^{2}\right. & \left.\left.+\left\|\boldsymbol{U}^{n}\right\|^{2}+\| \boldsymbol{U}^{n+1}-\boldsymbol{U}^{n}\right) \|^{2}\right)+\left(\left\|V^{n+1}\right\|^{2}-\left\|V^{n}\right\|^{2}+\left\|V^{n+1}-V^{n}\right\|^{2}\right) \\
& =-\delta t M\left\|\nabla \mu^{n+1}\right\|^{2} .
\end{aligned}
$$

Finally, we obtain the desired result (3.35) after dropping some positive terms.

Several remarks are in order.

Remark 3.2. The proposed scheme follows the new energy dissipation law (3.14) instead of the energy law for the originated system (2.15). For time-continuous case, (3.14) and (2.15) are identical. For time-discrete case, the discrete energy law (3.35) is the first order approximation to the new energy law (3.14). Moreover, the discrete energy functional $E_{1}\left(\boldsymbol{U}^{n+1}, V^{n+1}\right)$ is also the first order approximation to $E\left(\phi^{n+1}\right)$ (defined in (2.16)), since $\boldsymbol{U}^{n+1}, V^{n+1}$ are the first order approximations to $X^{n+1} \phi^{n+1}$ and $\sqrt{F\left(\phi^{n+1}\right)+B}$ respectively, that can be observed from the following facts, heuristically. We rewrite (3.19) as follows,

$$
\boldsymbol{U}^{n+1}-X^{n+1} \nabla \phi^{n+1}=\boldsymbol{U}^{n}-X^{n} \nabla \phi^{n}+R_{n+1},
$$

where

$$
R_{n+1}=-X^{n+1} \nabla \phi^{n+1}+X^{n} \nabla \phi^{n}+X^{n} \nabla\left(\phi^{n+1}-\phi^{n}\right)+\frac{1}{2} Y^{n}\left(\phi^{n+1}-\phi^{n}\right) \nabla \phi^{n} .
$$

Since $R_{n+1}$ is the resudue of the Taylor expansion for the function $X^{n+1} \nabla \phi^{n+1}$ at $t=t^{n}$, thus $R_{n+1}=O\left(\delta t^{2}\right)$ for $0 \leq k \leq n+1$. Note that $\boldsymbol{U}^{0}=X^{0} \nabla \phi^{0}$, by mathematical induction we can easily get $\boldsymbol{U}^{n+1}=X^{n+1} \nabla \phi^{n+1}+O(\delta t)$. The same argument can be performed for the other variable $V^{n+1}$. Actually, in other words, (3.17) and (3.18) are the Tylor expansion formulation for $\boldsymbol{U}^{n+1}$ and $V^{n+1}$ at $t=t^{n}$, respectively.

Remark 3.3. In [29], Qiao, Sun and Zhang proposed an approach to disretize the nonlinear Molecular beam epitaxial model. Their approach is quite similar to the IEQ approach in this paper but with some essential differences.

We list their procedure here in details. Suppose the free energy $E(\phi)=\int_{\Omega} F(\phi) d \boldsymbol{x}$ with $F(\boldsymbol{x})>$ $0, \forall \boldsymbol{x}$. The PDE system with $L^{2}$ gradient flow reads as follows,

$$
\phi_{t}=-f(\phi)
$$

where $f(\phi)=F^{\prime}(\phi)$. They define an auxillary variable $V=F(\phi)$, thus the PDE system turns to an equivalent system as follows

$$
\begin{aligned}
\phi_{t} & =-f(\phi), \\
V_{t} & =f(\phi) \phi_{t} .
\end{aligned}
$$

The new system still follows the PDE energy law by taking the $L^{2}$ inner product of the first equation 
in (3.46) with $\phi_{t}$ and applying the second equation, i.e.,

$$
\frac{d}{d t} E(V)=-\left\|\phi_{t}\right\|^{2}
$$

with

$$
E(V)=\int_{\Omega} V d \boldsymbol{x}
$$

This approach is quite similar to the idea of IEQ approach. The only difference is $V=F(\phi)$ (in [29]) and $V^{2}=F(\phi)$ (in our IEQ approach).

First, we notice that the new variable $V$ in [29] is not involved in the computations of $\phi$. Second, although the system (3.46) is equivalent to the original system (3.45), the free energy functional $E(V)$ in terms of the linear form of new variable $V$ may not be bounded from below. Therefore, this approach and its apparent energy stability condition (3.47) are not reliable. In contrast, in the IEQ approach, the quadratic formulation of $E(V)=\int_{\Omega} V^{2} d \boldsymbol{x}$ is naturally bounded from below.

Remark 3.4. The essential idea of the IEQ method is to transform the complicated nonlinear potentials into a simple quadratic form in terms set of some new variables via a change of variables. This idea is inspired by the work in [18, 19,35, 37], where the similar quadratization method had been applied for the polynomial type potentials for various phase field models. Overall, the IEQ approach provides many flexibilities to treat the complicated nonlinear terms, for instances, the nonlinear potential only need to be positive, or bounded from below, instead of the convexity that is required in the convex splitting approach (cf. [13]) and the boundness for the second order derivative in the stabilization approach (cf. [5, 31,34]).

Such a simple way of quadratization provides some great advantages. First, the complicated nonlinear potential is transferred to a quadratic polynomial form which is much easier to handle. Second, the derivative of the quadratic polynomial is linear, which provides the fundamental support for linearization method. Third, the quadratic formulation in terms of new variables can automatically keep this property of positivity (or bounded from below) of the nonlinear potentials.

Meanwhile, the choices of new variables are not unique. For instance, for the gradient entropy term in the PF-HB model, an alternative scalar function $\widehat{U}$ can be defined as follows,

$$
\widehat{U}(\phi)=\sqrt{\kappa(\phi)|\nabla \phi|^{2}+\widehat{B}}
$$

where $\widehat{B}$ is any postive constant which is used to ensure $\kappa(\phi)|\nabla \phi|^{2}+\widehat{B}>0$ (B can be any positive constant). Thus the free energy becomes

$$
E(\widehat{U}, V)=\int_{\Omega}\left(\epsilon^{2} \widehat{U}^{2}+V^{2}-B-\epsilon^{2} \widehat{B}\right) d \boldsymbol{x},
$$

and the equivalent PDE system becomes

$$
\begin{aligned}
& \phi_{t}=M \Delta \mu, \\
& \mu=\widehat{X}(\phi) \widehat{U}+Z(\phi) V, \\
& \widehat{U}_{t}=\widehat{X}(\phi) \phi_{t}, \\
& V_{t}=\frac{1}{2} Z(\phi) \phi_{t},
\end{aligned}
$$


where

$$
\widehat{X}(\phi)=\frac{-2 \epsilon^{2} \nabla \cdot(\kappa(\phi) \nabla \phi)+\epsilon^{2} \kappa^{\prime}(\phi)|\nabla \phi|^{2}}{\sqrt{\kappa(\phi)|\nabla \phi|^{2}+\widehat{B}}} .
$$

Note the new variable $\widehat{U}$ is now a scalar function instead of the vector function in (3.6) adpoted in this paper. As can be seen in the following, the IEQ approach is able to provide enough flexibilities to derive the equivalent PDE system, and hence the corresponding numerical schemes with unconditional energy stabilities. The developments of similar algorithms for the alternative system (3.51)-(3.54) are left to the interested readers.

Remark 3.5. The IEQ approach provides more efficiency than the nonlinear approach. For instances, when the IEQ approach is applied to the classical CH equation, we can have the linear scheme as follows,

$$
\begin{aligned}
& \frac{\phi^{n+1}-\phi^{n}}{\delta t}=\Delta \mu^{n+1}, \\
& \mu^{n+1}=-\Delta \phi^{n+1}+2\left(\phi^{n}\right)^{2} \phi^{n+1}+\phi^{n}\left(U^{n}-2\left(\phi^{n}\right)^{2}\right) .
\end{aligned}
$$

In contrast, we list the first order convex-splitting scheme [13] here,

$$
\begin{aligned}
& \frac{\phi^{n+1}-\phi^{n}}{\delta t}=\Delta \mu^{n+1}, \\
& \mu^{n+1}=-\Delta \phi^{n+1}+\left(\phi^{n+1}\right)^{3}-\phi^{n} .
\end{aligned}
$$

If the Newton iterative method is applied for solving the above nonlinear system (3.57), at each iteration it would yield the same linear system (3.56). Hence the cost of solving the scheme (3.56) using the IEQ approach is the same as the cost of performing one iteration of Newton method for the nonlinear scheme, provided that the same linear solvers are applied (for instance multi-grid with Gauss-Seidel relaxation). It is clear that the IEQ scheme (3.56) would be much more efficient than the nonlinear convex-splitting schemes.

Remark 3.6. For the numerical schemes proposed in this paper, the energy stability is formally derived. About the error estimates for the proposed numerical schemes when using the IEQ approach, things become a little bit complicated. For instances, when the IEQ approach is applied for the regular Cahn-Hilliard type equation where the coefficient of the gradient entropy is a constant, one can easily obtain $\mathrm{H}^{1}$ bound from the Poincare inequality, and the corresponding convergence analysis can be further carried out. However, for Allen-Cahn dynamics equation, we immediately notice that the uniform $H^{1}$ bound cannot be justified theoretically since the double well potential is replaced by a new variable. For the PF-HB model considered in this paper, we can still obtain the optimal error since the gradient entropy is totally replaced by the new variable. But the error bound is naturally the $L^{2}$ error in terms of the new variable $\boldsymbol{U}$ instead of the $H^{1}$ error for the original phase variable $\phi$. We will implement the rigorous error analaysis in the future work that will follow the same lines as the literatures $[10,11,14,31]$.

3.3. Second order Scheme. We now consider develop second-order scheme where the time derivative is discretized using the adam-Bashforth (BDF2). Having computed $(\phi, \boldsymbol{U}, V)^{n}$ and $(\phi$, $\boldsymbol{U}, V)^{n-1}$, we compute $\phi^{n+1}, \boldsymbol{U}^{n+1}, V^{n+1}$ as follows. 


$$
\begin{aligned}
& \frac{3 \phi^{n+1}-4 \phi^{n}+\phi^{n-1}}{2 \delta t}=M \Delta \mu^{n+1}, \\
& \mu^{n+1}=-2 \epsilon^{2} \nabla \cdot\left(X^{\star} \boldsymbol{U}^{n+1}\right)+\epsilon^{2} Y^{\star} \boldsymbol{U}^{n+1} \cdot \nabla \phi^{\star}+Z^{\star} V^{n+1}, \\
& 3 \boldsymbol{U}^{n+1}-4 \boldsymbol{U}^{n}+\boldsymbol{U}^{n-1}=X^{\star} \nabla\left(3 \phi^{n+1}-4 \phi^{n}+\phi^{n-1}\right)+\frac{1}{2} Y^{\star}\left(3 \phi^{n+1}-4 \phi^{n}+\phi^{n-1}\right) \nabla \phi^{\star}, \\
& 3 V^{n+1}-4 V^{n}+V^{n-1}=\frac{1}{2} Z^{\star}\left(3 \phi^{n+1}-4 \phi^{n}+\phi^{n-1}\right),
\end{aligned}
$$

where

$$
\phi^{\star}=2 \phi^{n}-\phi^{n-1}, X^{\star}=2 X^{n}-X^{n-1}, Y^{\star}=2 Y^{n}-Y^{n-1}, Z^{\star}=2 Z^{n}-Z^{n-1} .
$$

Similar to the first order scheme, we can rewrite the equations (3.60) and (3.61) as follows,

$$
\left\{\begin{array}{l}
\boldsymbol{U}^{n+1}=\mathbf{A}_{2}+X^{\star} \nabla \phi^{n+1}+\frac{1}{2} Y^{\star} \nabla \phi^{\star} \phi^{n+1}, \\
V^{n+1}=B_{2}+\frac{1}{2} Z^{\star} \phi^{n+1},
\end{array}\right.
$$

with

$$
\left\{\begin{array}{l}
\mathbf{A}_{2}=\boldsymbol{U}^{\dagger}-X^{\star} \nabla \phi^{\dagger}-\frac{1}{2} Y^{\star} \phi^{\dagger} \nabla \phi^{\star} \\
B_{2}=V^{\dagger}-\frac{1}{2} Z^{\star} \phi^{\dagger}
\end{array}\right.
$$

where $S^{\dagger}=\frac{4 S^{n}-S^{n-1}}{3}$ for any variable $S$. Thus, the system (3.58)- (3.61) can be rewritten as

$$
\begin{aligned}
& \frac{3 \phi^{n+1}-4 \phi^{n}+\phi^{n-1}}{2 \delta t}-M \Delta \mu^{n+1}=0, \\
& \mu^{n+1}-P_{2}\left(\phi^{n+1}\right)=Q_{2} .
\end{aligned}
$$

where

$$
\left\{\begin{aligned}
P_{2}(\phi)= & -2 \epsilon^{2} \nabla \cdot\left(X^{\star} X^{\star} \nabla \phi\right)-\epsilon^{2} \nabla \cdot\left(X^{\star} Y^{\star} \nabla \phi^{\star} \phi\right) \\
& +\epsilon^{2} X^{\star} Y^{\star} \nabla \phi^{\star} \nabla \phi+\frac{1}{2} \epsilon^{2} Y^{\star} Y^{\star} \nabla \phi^{\star} \cdot \nabla \phi^{\star} \phi+\frac{1}{2} Z^{\star} Z^{\star} \phi, \\
Q_{2}= & Z^{\star} B_{2}+\beta\left(1-2 \phi^{\star}\right)-2 \epsilon^{2} \nabla \cdot\left(X^{\star} \mathbf{A}_{2}\right)+\epsilon^{2} Y^{\star} \mathbf{A}_{2} \cdot \nabla \phi^{\star} .
\end{aligned}\right.
$$

Therefore, we can solve $\phi^{n+1}$ and $\mu^{n+1}$ directly from (3.65) and (3.66). Once we obtain $\phi^{n+1}$, the $\boldsymbol{U}^{n+1}, V^{n+1}$ are automatically given in (3.19). Namely, the new variables $\boldsymbol{U}, V$ do not involve any extra computational costs. Furthermore, like the first order scheme, we can easily derive

$$
\left(P_{2}(\phi), \psi\right)=\left(\phi, P_{2}(\psi)\right)
$$

for $\psi$ satisfies the boundary condition (2.10). Meanwhile, for any $\phi$ with mean zero, we have

$$
\left(P_{2}(\phi), \phi\right)=\epsilon^{2}\left\|\frac{1}{\sqrt{2}} Y^{\star} \nabla \phi^{\star} \phi+\sqrt{2} X^{\star} \nabla \phi\right\|^{2}+\frac{1}{2}\left\|Z^{\star} \phi\right\|^{2} \geq 0,
$$


where "=" is valid if and only if $\phi=0$.

THEOREM 3.3. The linear operator of the system of (3.65)-(3.66) is symmetric (self-adjoint) and postive definite for the variable $\phi^{n+1}$.

Proof. We omit the details here since the proof is similar to Theorem 3.1.

We prove the energy stability theorem as follows.

THEOREM 3.4. The scheme (3.58)-(3.61) is unconditionally stable, and admits a unique solution satisfying the following discrete energy dissipation law.

$$
\frac{1}{\delta t}\left(E_{2}^{n+1, n}-E_{2}^{n, n-1}\right) \leq-M\left\|\nabla \mu^{n+1}\right\|^{2}
$$

where

$$
E_{2}^{n+1, n}=\int_{\Omega}\left\{\epsilon^{2}\left(\frac{\left|\boldsymbol{U}^{n+1}\right|^{2}}{2}+\frac{\left|2 \boldsymbol{U}^{n+1}-\boldsymbol{U}^{n}\right|^{2}}{2}\right)+\left(\frac{\left(V^{n+1}\right)^{2}}{2}+\frac{\left(2 V^{n+1}-V^{n}\right)^{2}}{2}\right)-B\right\} d \boldsymbol{x} .
$$

Proof. By taking the $L^{2}$ inner product of (3.58) with $-2 \delta t \mu^{n+1}$, we obtain

$$
-\left(3 \phi^{n+1}-4 \phi^{n}+\phi^{n-1}, \mu^{n+1}\right)=2 \delta t M\left\|\nabla \mu^{n+1}\right\|^{2} .
$$

By taking the $L^{2}$ inner product of (3.59) with $3 \phi^{n+1}-4 \phi^{n}+\phi^{n-1}$, we derive

$$
\begin{aligned}
\left(3 \phi^{n+1}-4 \phi^{n}\right. & \left.+\phi^{n-1}, \mu^{n+1}\right) \\
= & 2 \epsilon^{2}\left(\boldsymbol{U}^{n+1} X^{\star}, \nabla\left(3 \phi^{n+1}-4 \phi^{n}+\phi^{n-1}\right)\right) \\
& +\epsilon^{2}\left(Y^{\star} \boldsymbol{U}^{n+1} \cdot \nabla\left(2 \phi^{n}-\phi^{n-1}\right), 3 \phi^{n+1}-4 \phi^{n}+\phi^{n-1}\right) \\
& +\left(Z^{\star} V^{n+1}, 3 \phi^{n+1}-4 \phi^{n}+\phi^{n-1}\right) .
\end{aligned}
$$

By taking the $L^{2}$ inner product of (3.60) with $-2 \epsilon^{2} \boldsymbol{U}^{n+1}$, and applying the following identity

$$
2(3 a-4 b+c, a)=\left(|a|^{2}+|2 a-b|^{2}\right)-\left(|b|^{2}+|2 b-c|^{2}\right)+|a-2 b+c|^{2},
$$

we obtain

$$
\begin{aligned}
-\epsilon^{2}\left(\left\|\boldsymbol{U}^{n+1}\right\|^{2}+\right. & \left.\left\|2 \boldsymbol{U}^{n+1}-\boldsymbol{U}^{n}\right\|^{2}\right) \\
& +\epsilon^{2}\left(\left\|\boldsymbol{U}^{n}\right\|^{2}-\left\|2 \boldsymbol{U}^{n}-\boldsymbol{U}^{n-1}\right\|^{2}\right)-\epsilon^{2}\left\|\boldsymbol{U}^{n+1}-2 \boldsymbol{U}^{n}+\boldsymbol{U}^{n-1}\right\|^{2} \\
= & -2 \epsilon^{2}\left(X^{\star} \nabla\left(3 \phi^{n+1}-4 \phi^{n}+\phi^{n-1}\right), \boldsymbol{U}^{n+1}\right) \\
& -\epsilon^{2}\left(Y^{\star}\left(3 \phi^{n+1}-4 \phi^{n}+\phi^{n-1}\right) \nabla\left(2 \phi^{n}-\phi^{n-1}\right), \boldsymbol{U}^{n+1}\right) .
\end{aligned}
$$

By taking the $L^{2}$ inner product of (3.61) with $-2 V^{n+1}$, we obtain

$$
\begin{aligned}
-\left(\left\|V^{n+1}\right\|^{2}+\left\|2 V^{n+1}-V^{n}\right\|^{2}\right) & +\left(\left\|V^{n}\right\|^{2}-\left\|2 V^{n}-V^{n-1}\right\|^{2}\right)-\left\|V^{n+1}-2 V^{n}+V^{n-1}\right\|^{2} \\
& =-\left(Z^{\star}\left(3 \phi^{n+1}-4 \phi^{n}+\phi^{n-1}\right), V^{n+1}\right) .
\end{aligned}
$$


By combining (3.72)-(3.73)-(3.75)-(3.76), we obtain

$$
\begin{aligned}
\epsilon^{2}\left(\frac{\left\|\boldsymbol{U}^{n+1}\right\|^{2}}{2}+\frac{\left\|2 \boldsymbol{U}^{n+1}-\boldsymbol{U}^{n}\right\|^{2}}{2}\right)-\epsilon^{2}\left(\frac{\left\|\boldsymbol{U}^{n}\right\|^{2}}{2}+\frac{\left\|2 \boldsymbol{U}^{n}-\boldsymbol{U}^{n-1}\right\|^{2}}{2}\right) \\
\quad+\left(\frac{\left\|V^{n+1}\right\|^{2}}{2}+\frac{\left\|2 V^{n+1}-V^{n}\right\|^{2}}{2}\right)-\left(\frac{\left\|V^{n}\right\|^{2}}{2}+\frac{\left\|2 V^{n}-V^{n-1}\right\|^{2}}{2}\right) \\
\quad+\epsilon^{2} \frac{\left\|\boldsymbol{U}^{n+1}-2 \boldsymbol{U}^{n}+\boldsymbol{U}^{n-1}\right\|^{2}}{2}+\frac{\left\|V^{n+1}-2 V^{n}+V^{n-1}\right\|^{2}}{2}=-M \delta t\left\|\nabla \mu^{n+1}\right\|^{2} .
\end{aligned}
$$

Finally, we obtain the desired result (3.70) after dropping some positive terms.

Remark 3.7. Heuristically, the discrete energy law (3.70) is a second order approximation of $\frac{d}{d t} E(\boldsymbol{U}, V)$ in (3.14) since

$$
\begin{aligned}
& \left(\frac{\left\|S^{n+1}\right\|^{2}+\left\|2 S^{n+1}-S^{n}\right\|^{2}}{2 \delta t}\right)-\left(\frac{\left\|S^{n}\right\|^{2}+\left\|2 S^{n}-S^{n-1}\right\|^{2}}{2 \delta t}\right) \\
& \cong\left(\frac{\left\|S^{n+2}\right\|^{2}-\left\|S^{n}\right\|^{2}}{2 \delta t}\right)+o\left(\delta t^{2}\right) \cong \frac{d}{d t}\left\|S\left(t^{n+1}\right)\right\|^{2}+O\left(\delta t^{2}\right) .
\end{aligned}
$$

One can easily develop the second order version of Crank-Nicolson scheme as follows,

$$
\begin{aligned}
& \frac{\phi^{n+1}-\phi^{n}}{\delta t}=M \Delta \mu^{n+\frac{1}{2}}, \\
& \mu^{n+\frac{1}{2}}=-2 \epsilon^{2} \nabla \cdot\left(X^{\circ} \boldsymbol{U}^{n+\frac{1}{2}}\right)+\epsilon^{2} Y^{\circ} \boldsymbol{U}^{n+\frac{1}{2}} \cdot \nabla \phi^{\circ}+Z^{\circ} V^{n+\frac{1}{2}}, \\
& \boldsymbol{U}^{n+1}-\boldsymbol{U}^{n}=X^{\circ} \nabla\left(\phi^{n+1}-\phi^{n}\right)+\frac{1}{2} Y^{\circ}\left(\phi^{n+1}-\phi^{n}\right) \nabla \phi^{\circ}, \\
& V^{n+1}-V^{n}=\frac{1}{2} Z^{\circ}\left(\phi^{n+1}-\phi^{n}\right),
\end{aligned}
$$

where

$$
\left\{\begin{array}{l}
\phi^{\circ}=\frac{3}{2} \phi^{n}-\frac{1}{2} \phi^{n-1}, \\
X^{\circ}=\frac{3}{2} X^{n}-\frac{1}{2} X^{n-1}, Y^{\circ}=\frac{3}{2} Y^{n}-\frac{1}{2} Y^{n-1}, Z^{\circ}=\frac{3}{2} Z^{n}-\frac{1}{2} Z^{n-1}, \\
\boldsymbol{U}^{n+\frac{1}{2}}=\frac{\boldsymbol{U}^{n+1}+\boldsymbol{U}^{n}}{2}, V^{n+\frac{1}{2}}=\frac{V^{n+1}+V^{n}}{2} .
\end{array}\right.
$$

Similar to the first order scheme, we can rewrite the equations (3.81) and (3.82) as follows,

$$
\left\{\begin{array}{l}
\boldsymbol{U}^{n+1}=\mathbf{A}_{3}+X^{\circ} \nabla \phi^{n+1}+\frac{1}{2} Y^{\circ} \nabla \phi^{\circ} \phi^{n+1}, \\
V^{n+1}=B_{3}+\frac{1}{2} Z^{\circ} \phi^{n+1}
\end{array}\right.
$$

with

$$
\left\{\begin{array}{l}
\mathbf{A}_{3}=\boldsymbol{U}^{n}-X^{\circ} \nabla \phi^{n}-\frac{1}{2} Y^{\circ} \phi^{n} \nabla \phi^{\circ}, \\
B_{3}=V^{n}-\frac{1}{2} Z^{\circ} \phi^{n} .
\end{array}\right.
$$


Therefore, the system (3.79)- (3.82) can be rewritten as

$$
\begin{aligned}
& \frac{\phi^{n+1}-\phi^{n}}{\delta t}-M \Delta \mu^{n+\frac{1}{2}}=0, \\
& \mu^{n+\frac{1}{2}}-P_{3}\left(\phi^{n+1}\right)=Q_{3} .
\end{aligned}
$$

where

$$
\left\{\begin{aligned}
P_{3}(\phi)= & -\epsilon^{2} \nabla \cdot\left(X^{\circ} X^{\circ} \nabla \phi\right)-\frac{1}{2} \epsilon^{2} \nabla \cdot\left(X^{\circ} Y^{\circ} \nabla \phi^{\circ} \phi\right) \\
& +\frac{1}{2} \epsilon^{2} X^{\circ} Y^{\circ} \nabla \phi^{\circ} \nabla \phi+\frac{1}{4} \epsilon^{2} Y^{\circ} Y^{\circ} \nabla \phi^{\circ} \cdot \nabla \phi^{\circ} \phi+\frac{1}{4} Z^{\circ} Z^{\circ} \phi \\
= & \frac{1}{2} Z^{\star} B_{3}+\beta\left(1-2 \phi^{\star}\right)-\epsilon^{2} \nabla \cdot\left(X^{\circ} \mathbf{A}_{3}+X^{\circ} \boldsymbol{U}^{n}\right)+\frac{1}{2} \epsilon^{2} Y^{\star}\left(\mathbf{A}_{3}+\boldsymbol{U}^{n}\right) \cdot \nabla \phi^{\circ} .
\end{aligned}\right.
$$

Therefore, we can solve $\phi^{n+1}$ and $\mu^{n+\frac{1}{2}}$ directly from (3.86) and (3.87). Once we obtain $\phi^{n+1}$, the $\boldsymbol{U}^{n+1}, V^{n+1}$ is automatically given in (3.84). Namely, the new variable $\boldsymbol{U}, V$ do not involve any extra computational costs. Furthermore, like the first order Euler scheme and the second order BDF2 scheme, we can easily derive

$$
\left(P_{3}(\phi), \psi\right)=\left(\phi, P_{3}(\psi)\right)
$$

for $\psi$ satisfies the periodic boundary condition or the no-flux boundary condition (2.10); and for any $\phi$ with mean zero,

$$
\left(P_{3}(\phi), \phi\right)=\frac{1}{2} \epsilon^{2}\left\|\frac{1}{\sqrt{2}} Y^{\circ} \nabla \phi^{\circ} \phi+\sqrt{2} X^{\circ} \nabla \phi\right\|^{2}+\frac{1}{4}\left\|Z^{\circ} \phi\right\|^{2} \geq 0,
$$

where "=" is valid if and only if $\phi=0$. Therefore, the linear operator $P_{3}(\phi)$ is symmetric (selfadjoint) postive definite.

THEOREM 3.5. The linear operator of the system of (3.79)-(3.82) is symmetric (self-adjoint) and postive definite.

Proof. We omit the details here since the proof is similar to Theorem 3.1.

THEOREM 3.6. The scheme (3.79)-(3.82) is unconditionally stable, and admits a unique solution satisfying the following discrete energy dissipation law.

$$
\frac{1}{\delta t}\left(E_{3}^{n+1}-E_{3}^{n}\right)=-M\left\|\nabla \mu^{n+\frac{1}{2}}\right\|^{2},
$$

where

$$
E_{3}^{n+1}=\int_{\Omega}\left(\epsilon^{2}\left|\boldsymbol{U}^{n+1}\right|^{2}+\left(V^{n+1}\right)^{2}-B\right) d \boldsymbol{x} .
$$

Proof. We omit the details here since the proof is similar to Theorem 3.2 and 3.4. We remark that the energy law is "=" instead of " $\leq$ ".

Remark 3.8. Although we consider only time discrete schemes in this paper, the results here can be carried over to any consistent finite-dimensional Galerkin type approximations since the analyses are based on the variational formulation with all test functions in the same space as the space of the trial functions. 


\begin{tabular}{c|c|c|c|c|c|c}
\hline$\delta t$ & LS1 & Order & LS2-BDF2 & Order & LS2-CN2 & Order \\
\hline \hline $2 \times 10^{-2}$ & $4.90 \mathrm{E}(-4)$ & - & $1.49 \mathrm{E}(-4)$ & - & $1.40 \mathrm{E}(-4)$ & - \\
\hline $1 \times 10^{-2}$ & $2.45 \mathrm{E}(-4)$ & 1.00 & $3.68 \mathrm{E}(-5)$ & 2.02 & $3.47 \mathrm{E}(-5)$ & 2.01 \\
\hline $5 \times 10^{-3}$ & $1.22 \mathrm{E}(-4)$ & 1.01 & $9.20 \mathrm{E}(-6)$ & 2.00 & $8.68 \mathrm{E}(-6)$ & 2.00 \\
\hline $2.5 \times 10^{-3}$ & $6.15 \mathrm{E}(-5)$ & 0.99 & $2.30 \mathrm{E}(-6)$ & 2.00 & $2.17 \mathrm{E}(-6)$ & 2.00 \\
\hline $1.25 \times 10^{-3}$ & $3.07 \mathrm{E}(-5)$ & 1.00 & $5.75 \mathrm{E}(-7)$ & 2.00 & $5.43 \mathrm{E}(-7)$ & 2.00 \\
\hline $6.25 \times 10^{-4}$ & $1.53 \mathrm{E}(-5)$ & 1.00 & $1.44 \mathrm{E}(-7)$ & 2.00 & $1.36 \mathrm{E}(-7)$ & 2.00 \\
\hline $3.125 \times 10^{-4}$ & $7.68 \mathrm{E}(-6)$ & 0.99 & $3.59 \mathrm{E}(-8)$ & 2.00 & $3.39 \mathrm{E}(-8)$ & 2.00 \\
\hline $1.5625 \times 10^{-4}$ & $3.84 \mathrm{E}(-6)$ & 1.00 & $8.98 \mathrm{E}(-9)$ & 2.00 & $8.48 \mathrm{E}(-9)$ & 2.00 \\
\hline $7.8125 \times 10^{-5}$ & $1.92 \mathrm{E}(-6)$ & 1.00 & $2.24 \mathrm{E}(-9)$ & 2.00 & $2.12 \mathrm{E}(-9)$ & 2.00 \\
\hline
\end{tabular}

TABLE 4.1

The $L^{2}$ numerical errors at $t=0.1$ that are computed by schemes LS1, LS2-BDF2 and LS2-CN2 using various temporal resolutions with the exact solution of (4.2). The order parameters are of (4.1) and $129^{2}$ Fourier modes are used to discretize the space.

\begin{tabular}{c|c|c|c|c|c|c}
\hline$\delta t$ & LS1 & Order & LS2-BDF2 & Order & LS2-CN2 & Order \\
\hline \hline $1 \times 10^{-2}$ & $2.10 \mathrm{E}(-4)$ & - & $5.01 \mathrm{E}(-5)$ & - & $4.98 \mathrm{E}(-5)$ & - \\
\hline $5 \times 10^{-3}$ & $1.08 \mathrm{E}(-4)$ & 0.96 & $1.38 \mathrm{E}(-5)$ & 1.86 & $1.35 \mathrm{E}(-5)$ & 1.88 \\
\hline $2.5 \times 10^{-3}$ & $5.52 \mathrm{E}(-5)$ & 0.97 & $3.63 \mathrm{E}(-6)$ & 1.93 & $3.51 \mathrm{E}(-6)$ & 1.94 \\
\hline $1.25 \times 10^{-3}$ & $2.79 \mathrm{E}(-5)$ & 0.98 & $9.27 \mathrm{E}(-7)$ & 1.87 & $9.18 \mathrm{E}(-7)$ & 1.93 \\
\hline $6.25 \times 10^{-4}$ & $1.40 \mathrm{E}(-5)$ & 0.99 & $2.31 \mathrm{E}(-7)$ & 2.10 & $2.19 \mathrm{E}(-7)$ & 2.07 \\
\hline $3.125 \times 10^{-4}$ & $7.02 \mathrm{E}(-6)$ & 1.00 & $5.58 \mathrm{E}(-8)$ & 2.05 & $5.28 \mathrm{E}(-8)$ & 2.05 \\
\hline $1.5625 \times 10^{-4}$ & $3.51 \mathrm{E}(-6)$ & 1.00 & $1.23 \mathrm{E}(-8)$ & 2.18 & $1.33 \mathrm{E}(-8)$ & 1.99 \\
\hline $7.8125 \times 10^{-5}$ & $1.74 \mathrm{E}(-6)$ & 1.01 & $2.91 \mathrm{E}(-9)$ & 2.08 & $3.18 \mathrm{E}(-9)$ & 2.06 \\
\hline
\end{tabular}

TABLE 4.2

The $L^{2}$ numerical errors at $t=0.5$ that are computed by schemes LS1, LS2-BDF2 and LS2-CN2 using various temporal resolutions with the initial conditions of (4.3), for mesh refinement test in time. The order parameters are of (4.1) and $129^{2}$ Fourier modes are used to discretize the space.

\section{Numerical Examples.}




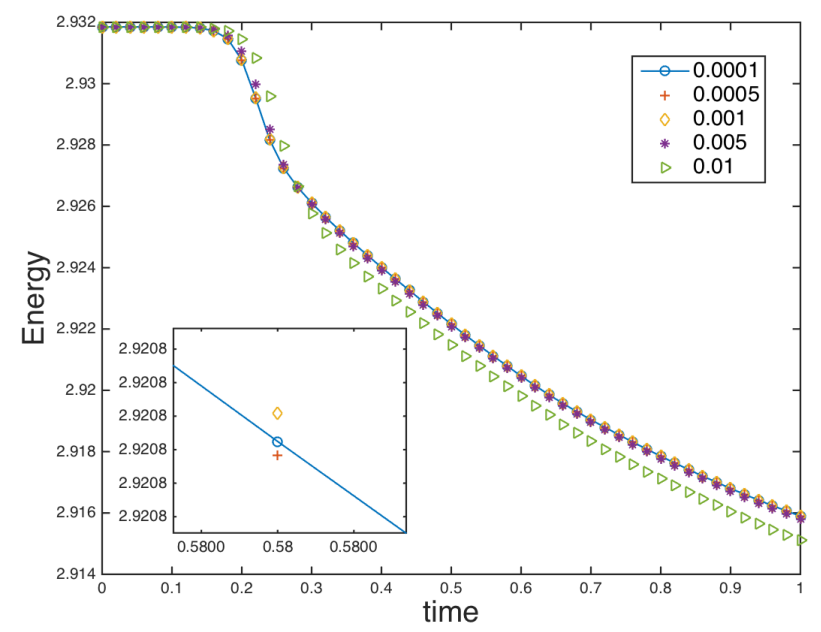

FIG. 4.1. Time evolution of the free energy functional for five different time steps of $\delta t=0.0001,0.0005,0.001$, 0.005 , and 0.01 for $\phi_{0}=0.3$. The energy curves show the decays for all time steps, which confirms that our algorithm is unconditionally stable. The small inset figure shows the small differences in the energy evolution for all four time steps.
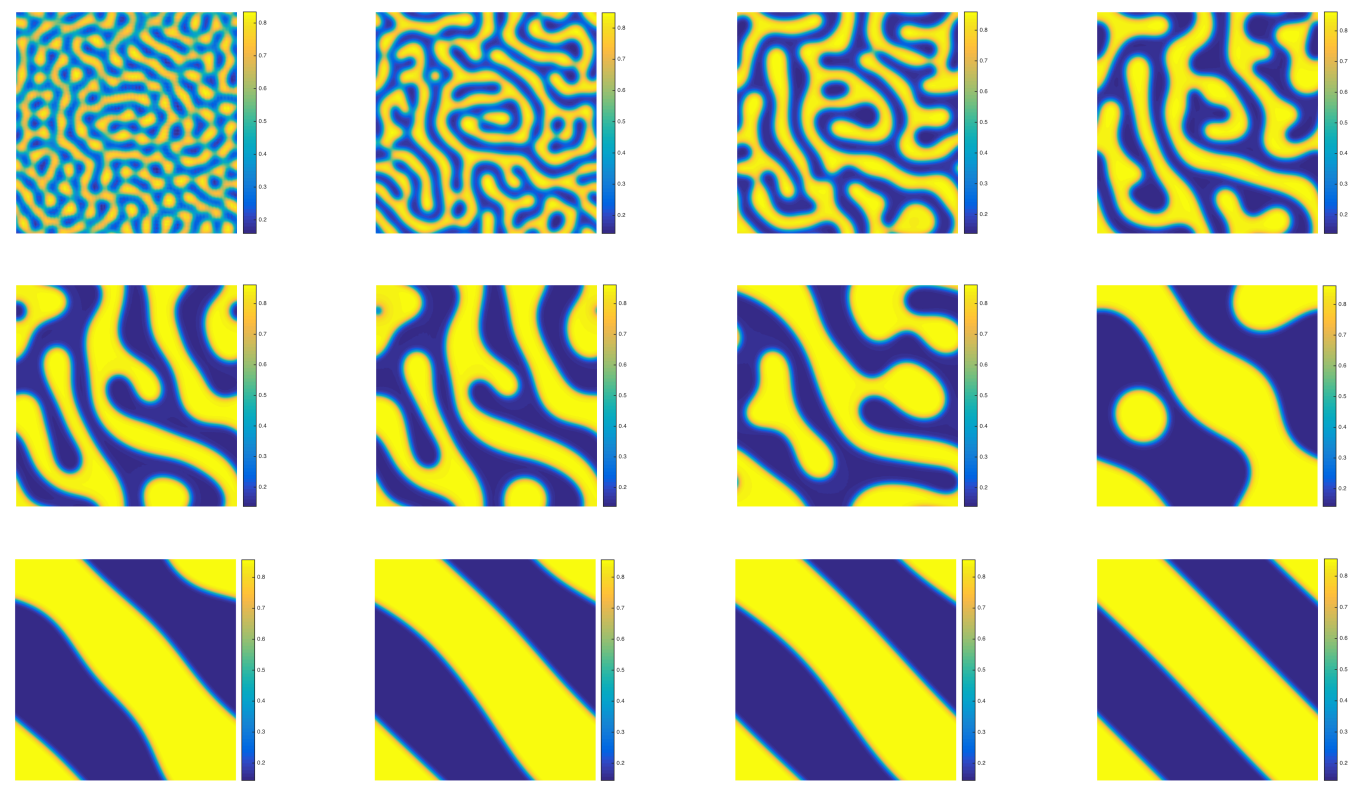

FIG. 4.2. The 2D dynamical evolution of the local volume fraction phase variable $\phi$ with the initial condition $\bar{\phi}_{0}=0.5$ and time step $\delta t=0.001$. Snapshots of the numerical approximation are taken at $t=0.25,0.5,1,2.5,3$, 5, 20, 40, 60, 80 and 200. 

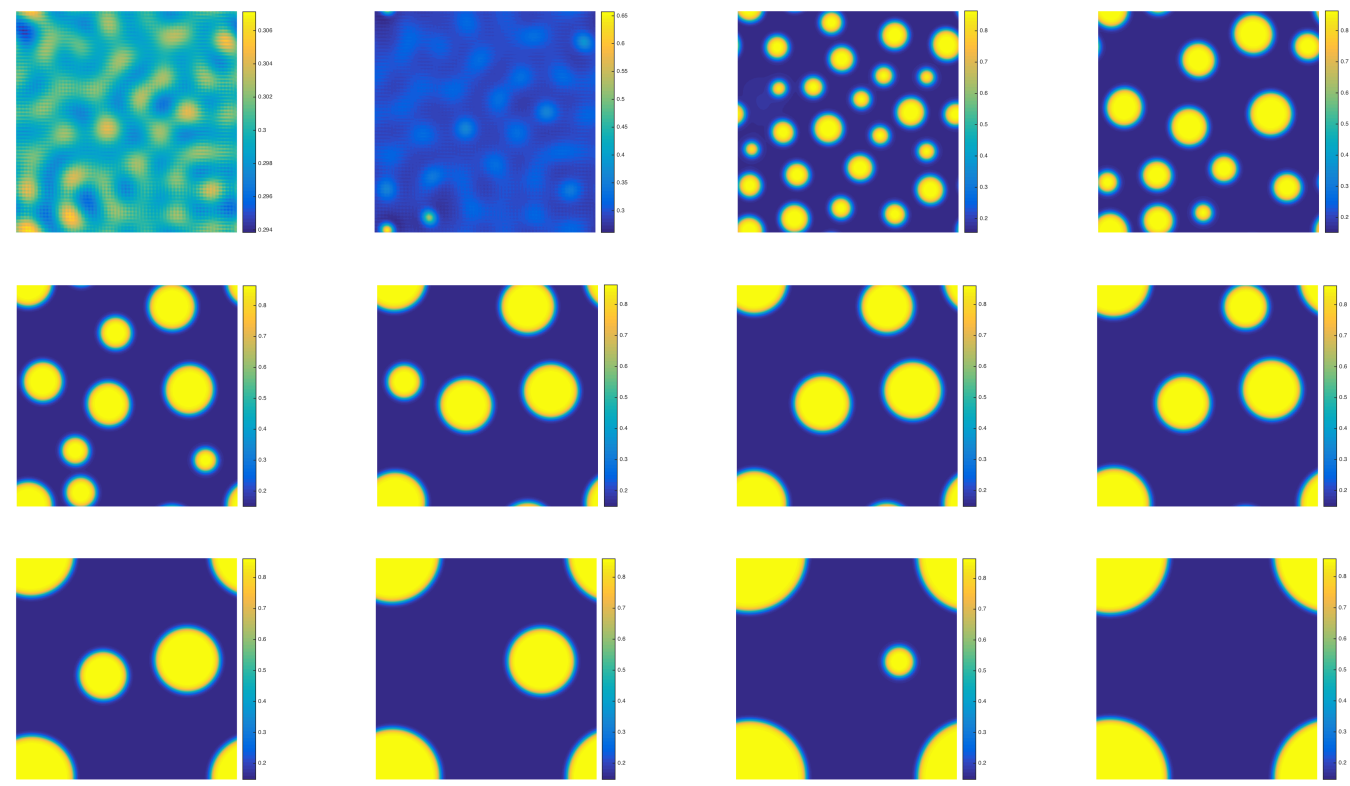

FIG. 4.3. The 2D dynamical evolution of the local volume fraction phase variable $\phi$ with the initial condition $\bar{\phi}_{0}=0.3$ and time step $\delta t=0.001$. Snapshots of the numerical approximation are taken at $t=1,1.5,2.5,5,10,20$, $30,50,70,100,150$ and 200.
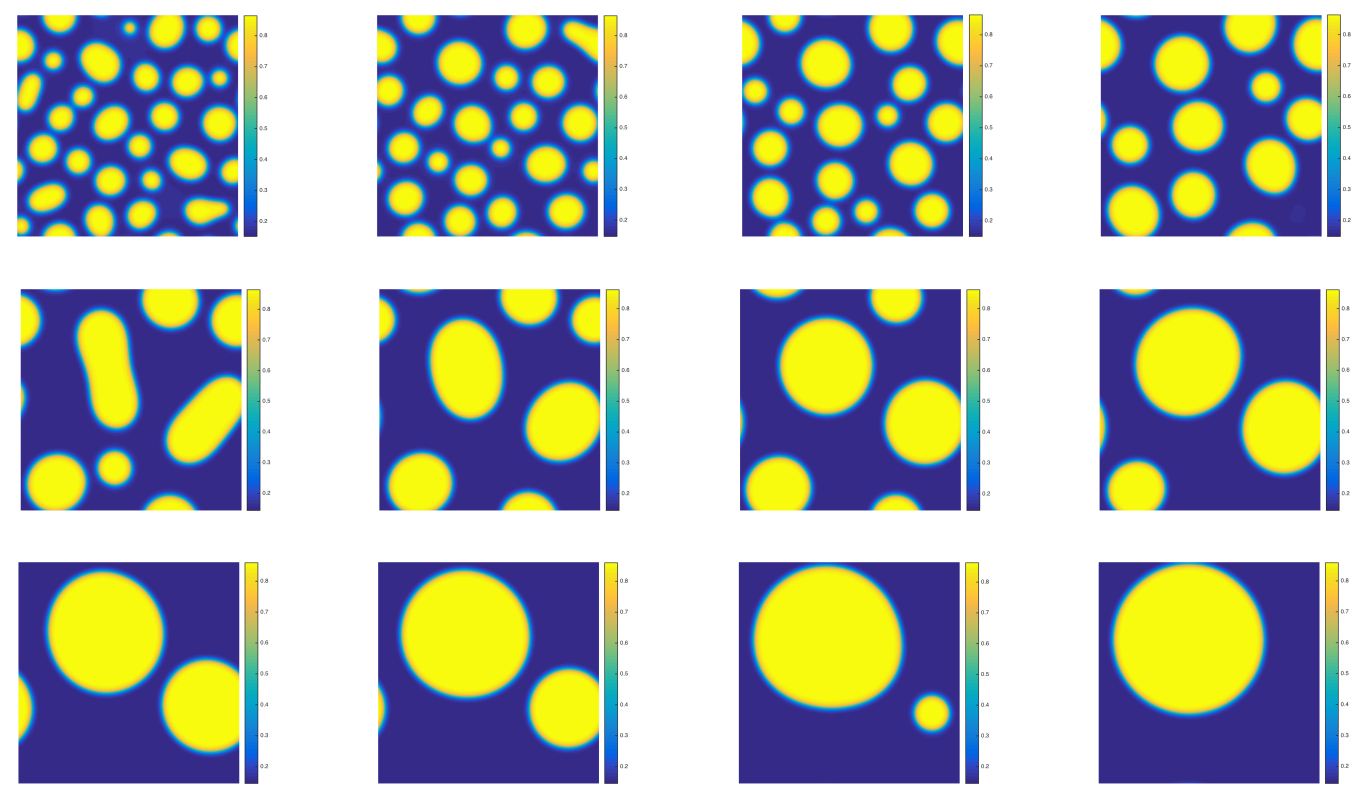

FIG. 4.4. The 2D dynamical evolution of the local volume fraction phase variable $\phi$ with the initial condition $\bar{\phi}_{0}=0.4$ and time step $\delta t=0.001$. Snapshots of the numerical approximation are taken at $t=1.5,2.5,5,10,20$, $30,50,70,100,150,200$ and 300 . 


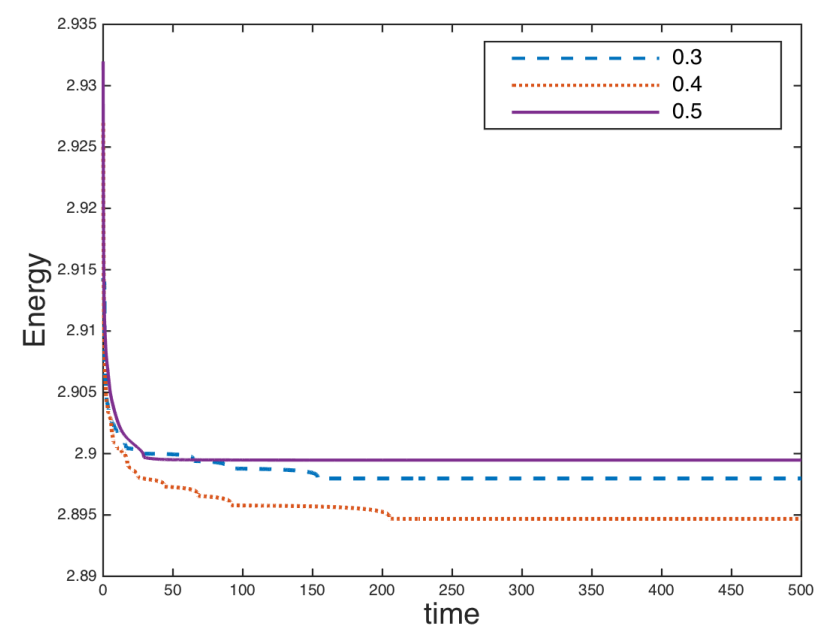

FIG. 4.5. Time evolution of the free energy functional for all three initial conditions of $\phi=0.3,0.4,0.5$ for the $2 D$ examples. The energy curves show the decays for all time steps, which confirms that our algorithm is unconditionally stable. The order parameters are of (4.1) and the time step is $\delta t=0.001$.
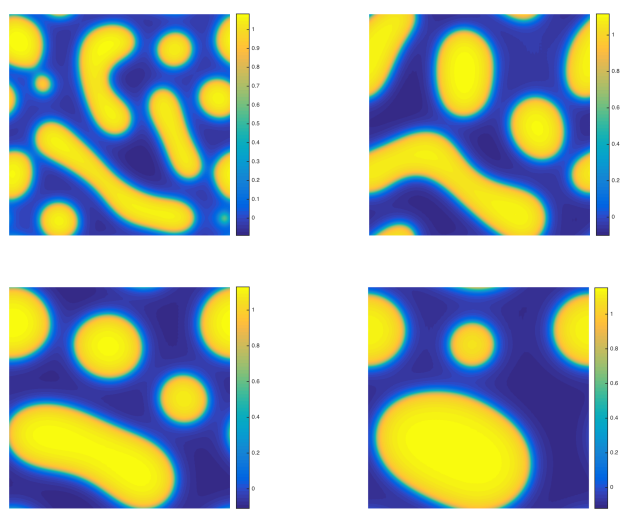
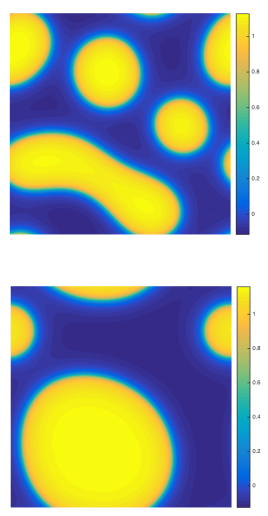

FIG. 4.6. The 2D dynamical evolution of the local volume fraction phase variable $\phi$ with the initial condition $\bar{\phi}_{0}=0.4$ and time step $\delta t=0.001$, where $\beta=5.5$. Snapshots of the numerical approximation are taken at $t=0.3$, $0.6,1,1.5,3,5$.

We now present various numerical experiments in $2 \mathrm{D}$ and $3 \mathrm{D}$ to validate the theoretical results derived in the previous section and demonstrate the efficiency, energy stability and accuracy of the proposed numerical schemes. In all examples, we use the periodic boundary conditions and the Fourier-Spectral method to discretize the computational domain $\Omega=[0,2 \pi]^{d}, d=2,3$. We use $129^{d}$ Fourier modes so that the errors from the spatial discretization are negligible compared with the time discretization errors. If not explicitly specified, the default values of order parameters are given as follows,

$$
\epsilon=0.02, M=1, \beta=2.5, \sigma=0.0001 \text {. }
$$



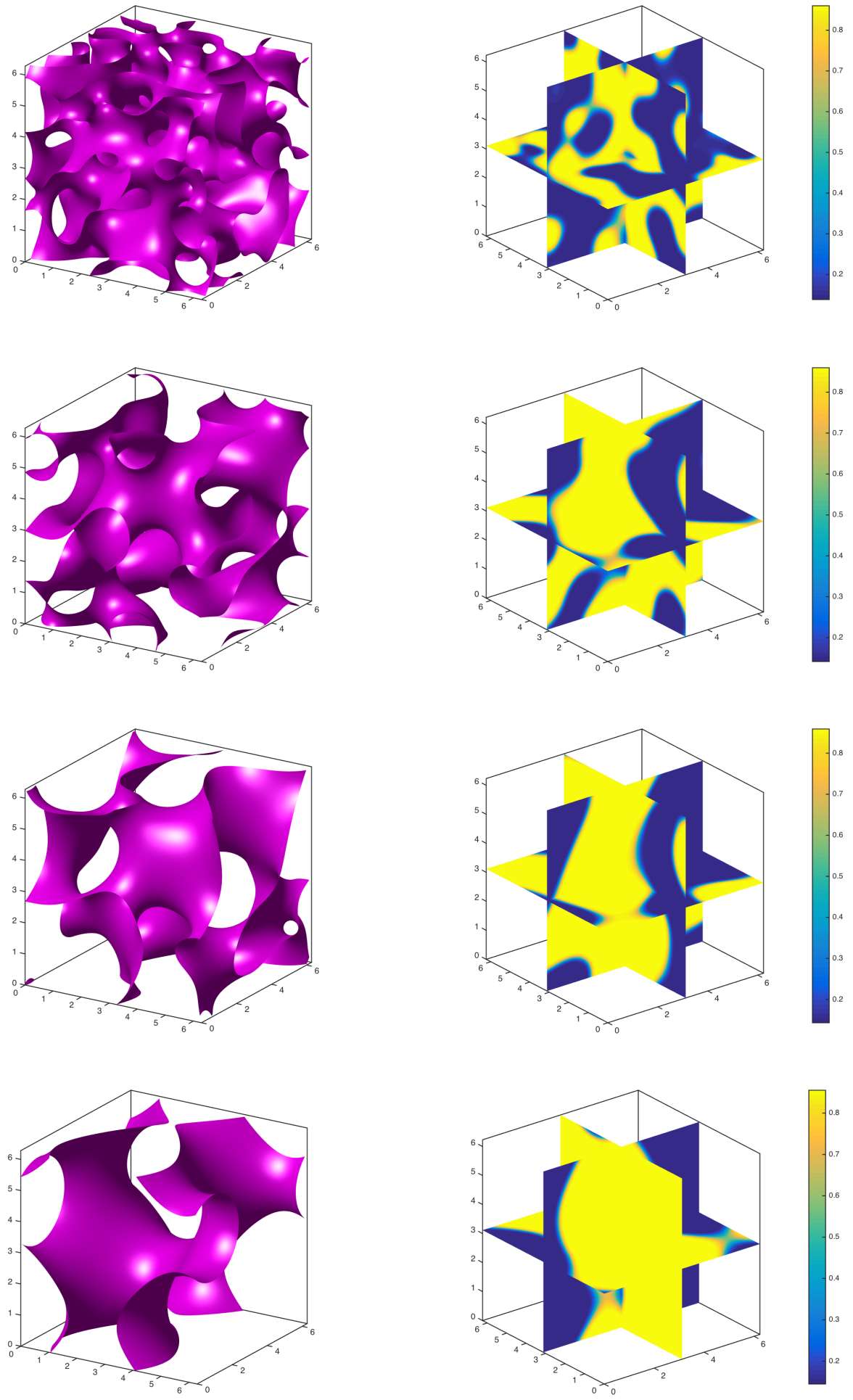

FIG. 4.7. The 3D dynamical evolution of the local volume fraction phase variable $\phi$ with the initial condition $\bar{\phi}_{0}=0.5$ and time step $\delta t=0.001$. Snapshots of the numerical approximation are taken at $t=10,40,80,500$. The left panel is the isosurface of the level set $\{\phi=0.5\}$, and the right panel is the three slices of the solutions across the indicated plane. 

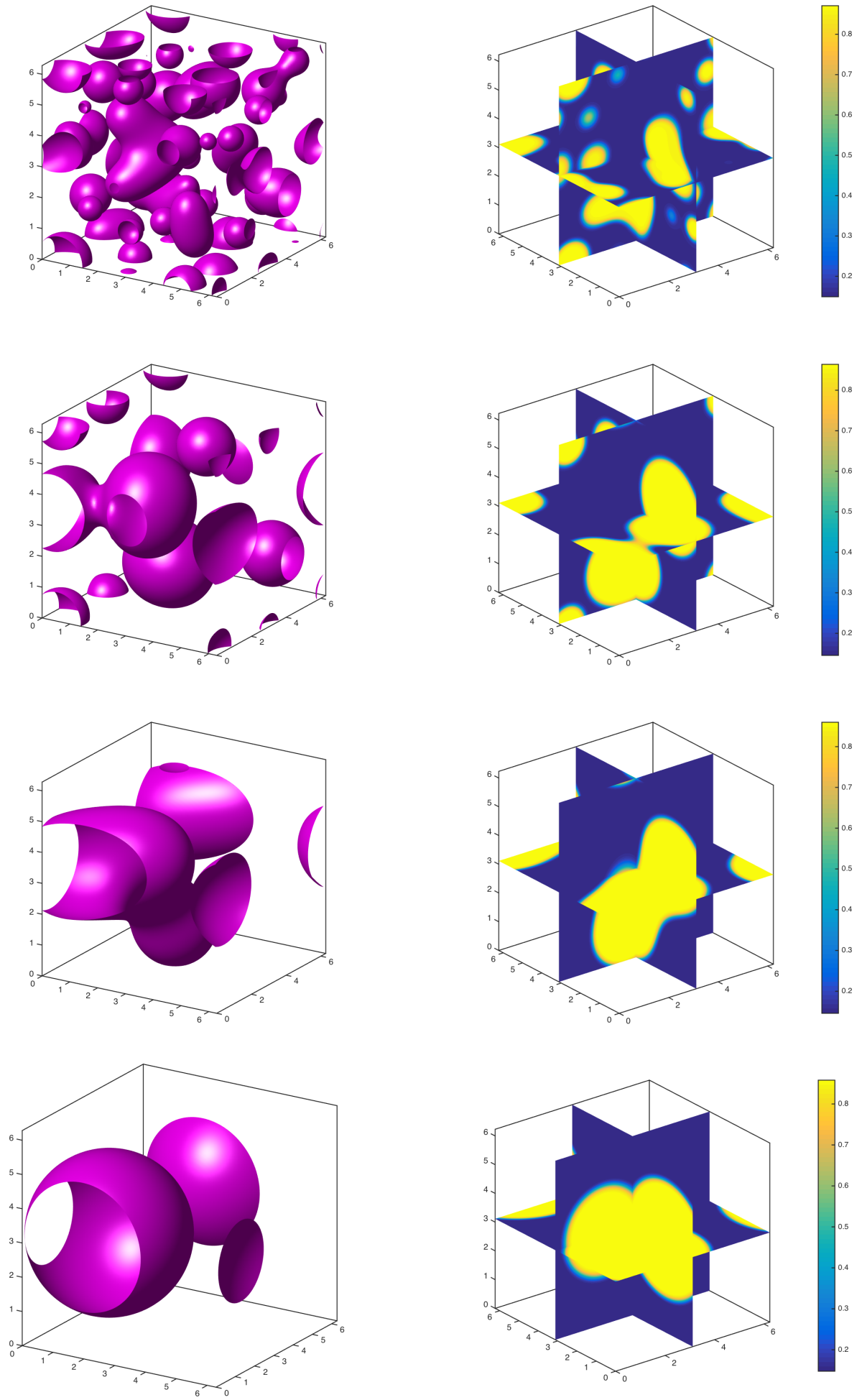

FIG. 4.8. The 3D dynamical evolution of the local volume fraction phase variable $\phi$ with the initial condition $\bar{\phi}_{0}=0.3$ and time step $\delta t=0.001$. Snapshots of the numerical approximation are taken at $t=10,40,80,500$. The left panel is the isosurface of the level set $\{\phi=0.5\}$, and the right panel is the three slices of the solutions across the indicated plane. 

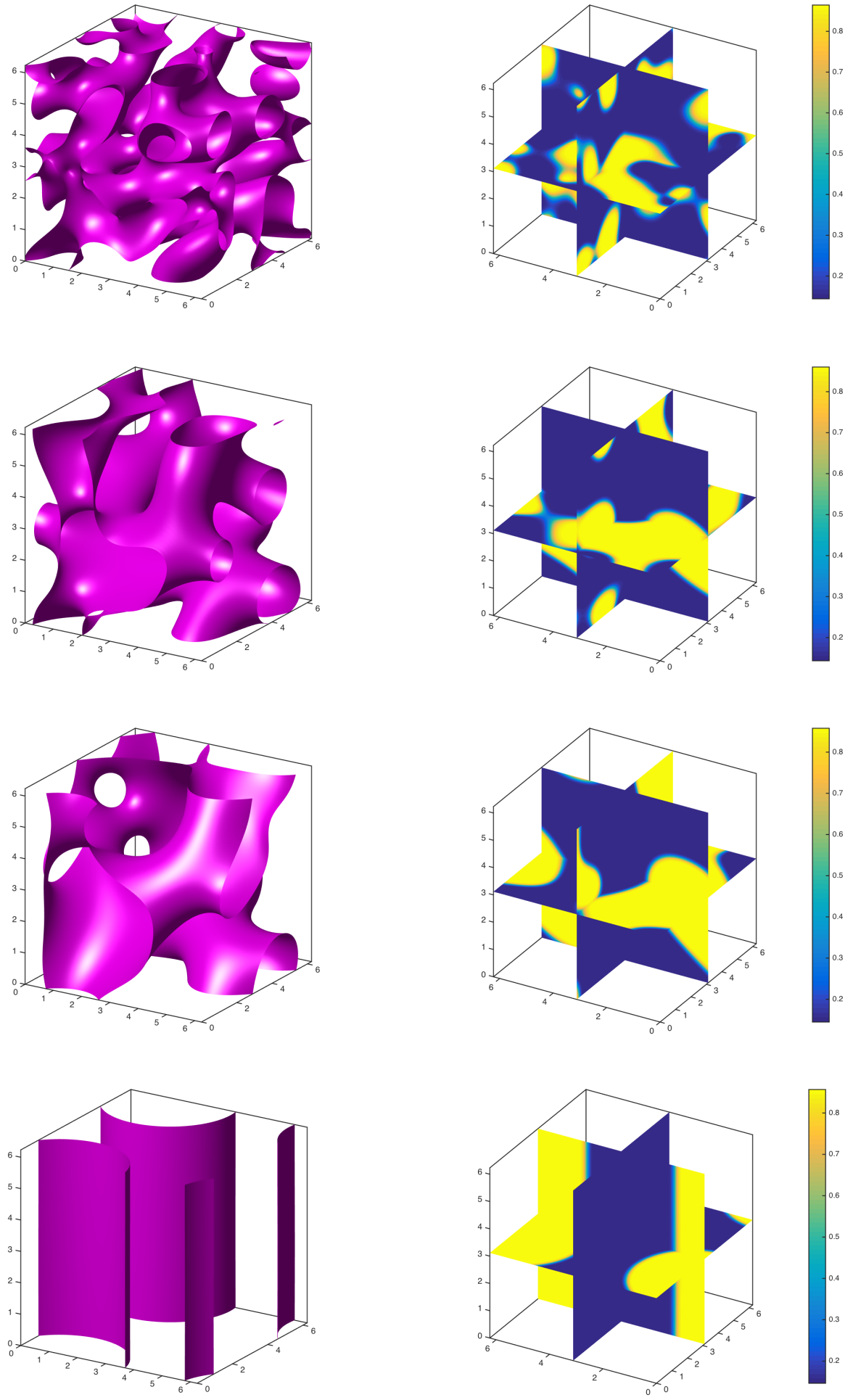

FIG. 4.9. The 3D dynamical evolution of the local volume fraction phase variable $\phi$ with the initial condition $\bar{\phi}_{0}=0.4$ and time step $\delta t=0.001$. Snapshots of the numerical approximation are taken at $t=10,40,80,500$. The left panel is the isosurface of the level set $\{\phi=0.5\}$, and the right panel is the three slices of the solutions across the indicated plane. 

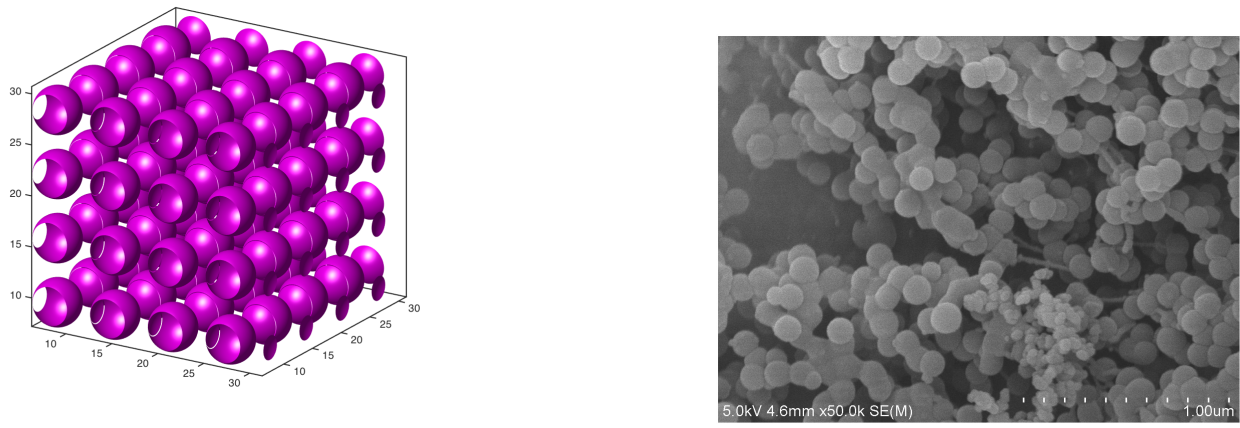

(a) $\phi_{0}=0.3$
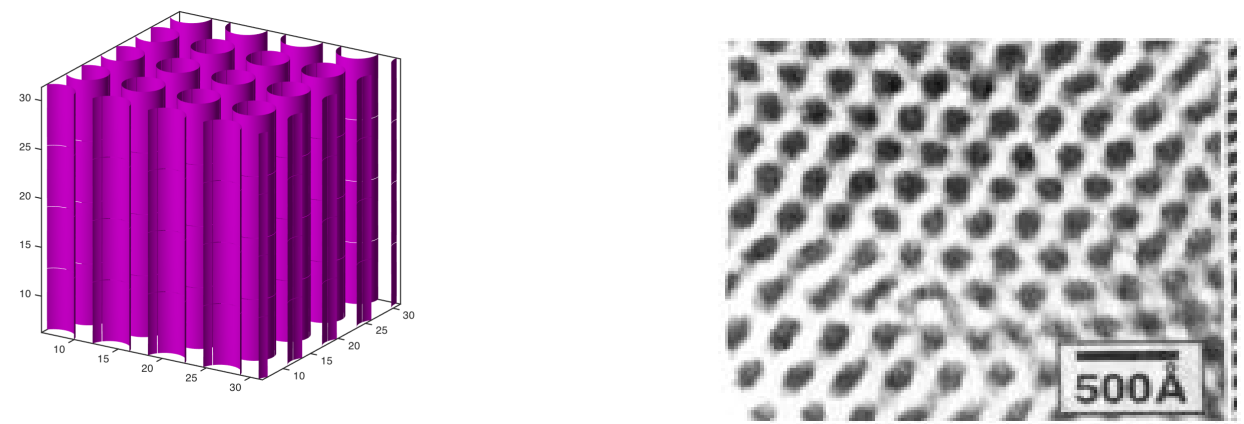

(b) $\phi_{0}=0.4$
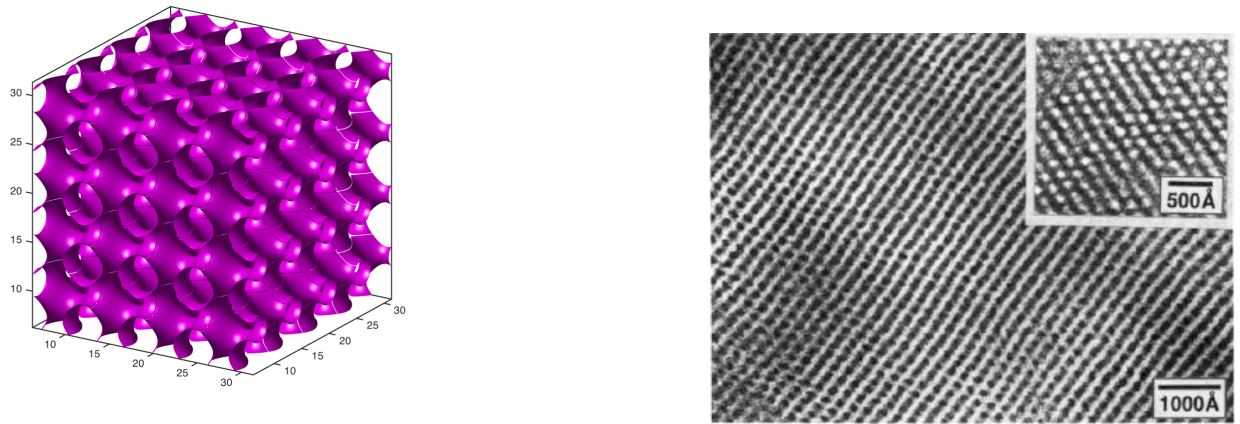

(c) $\phi_{0}=0.5$.

FIG. 4.10. The comparisons of isosurfaces of the equilibrium solutions for three initial values of $\bar{\phi}_{0}=0.3,0.4,0.5$, with the experiment results from [17, 36]. 


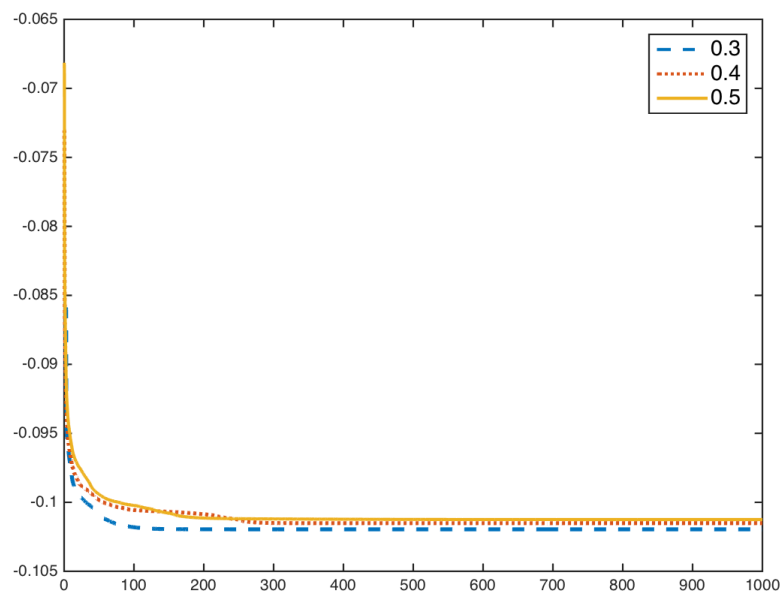

FIG. 4.11. Time evolution of the free energy functional for all three initial conditions of $\phi=0.3,0.4,0.5$ for the 3D examples. The energy curves show the decays for all time steps, which confirms that our algorithm is unconditionally stable. The order parameters are of (4.1) and the time step is $\delta t=0.001$.

4.1. Accuray test. We first test the convergence rates of the three proposed schemes in 2D. We denote the first order Euler scheme (3.15)-(3.18) by LS1, the second order BDF2 scheme (3.58)(3.61) by LS2-BDF2, and the second order Crank-Nicolson scheme (3.79)-(3.82) by LS2-CN2.

4.1.1. Presumed exact solution. In this example, we assume the following function

$$
\phi(x, y, t)=\left(\frac{\sin (2 x) \sin (2 y)}{4}+0.48\right)\left(1-\frac{\sin ^{2}(t)}{2}\right)
$$

to be the exact solution, and impose some suitable force fields such that the given solution can satisfy the system (2.7)-(2.8). In Table 4.1, we list the $L^{2}$ errors of the phase variable between the numerical solution and the exact solution at $t=0.1$ with different time step sizes. We observe that the schemes LS1, LS2-BDF2 and LS2-CN2 almost perfectly match the first-order and second order accuracy in time, respectively.

4.1.2. Mesh refinement in time. We now perform more refinement tests for temporal convergence. We set the initial conditions as follows,

$$
\phi(0, x, y)=\frac{\sin (2 x) \cos (2 y)}{4}+0.48
$$

We perform the refinement test of the time step size. Since the exact solutions are not known, we choose the solution obtained by the scheme LS2-BDF2 with the time step size $\delta t=1.0 \times 10^{-6}$ as the benchmark solution (approximately the exact solution) for computing errors. We present the $L^{2}$ errors of the phase variable between the numerical solution and the exact solution at $t=0.1$ with different time step sizes in Table 4.2. We observe that the schemes LS1, LS2-BDF2 and LS2-CN2 asymptotically match the first-order and second order accuracy in time, respectively. Moreover, the two second order scheme gives better accuracy than the first order scheme LS1 does when using the same time step. 
4.2. Spinodal decomposition in $2 \mathrm{D}$. In this example, we study the phase separation dynamics that is called spinodal decomposition using the second order scheme LS-BDF2. The process of the phase separation can be studied by considering a homogeneous binary mixture, which is quenched into the unstable part of its miscibility gap. In this case, the spinodal decomposition takes place, which manifests in the spontaneous growth of the concentration fluctuations that leads the system from the homogeneous to the two-phase state. Shortly after the phase separation starts, the domains of the binary components are formed and the interface between the two phases can be specified $[1,9,38]$.

The initial condition is taken as the randomly perturbed concentration field as follows,

$$
\phi(t=0)=\bar{\phi}_{0}+0.001 \operatorname{rand}(x, y),
$$

where the $\operatorname{rand}(x, y)$ is the random number in $[-1,1]$ that follows the normal distribution.

We emphasize that any time step size $\delta t$ is allowable for the computations from the stability concern since all developed schemes are unconditionally energy stable. But larger time step will definitely induce large numerical errors. Therefore, we need to discover the rough range of the allowable maximum time step size in order to obtain good accuracy and to consume as low computational cost as possible. This time step range could be estimated through the energy evolution curve plots, shown in Fig. 4.1, where we compare the time evolution of the free energy for five different time step sizes until $t=1$ using the second order scheme LS2-BDF2. We observe that all five energy curves show decays monotonically for all time step sizes, which numerically confirms that our algorithms are unconditionally energy stable. For smaller time steps of $\delta t=0.0001,0.0005,0.001,0.005$, the four energy curves coincide very well. But for the larger time step of $\delta t=0.01(\sim O(\epsilon))$, the energy curve deviates viewable away from others. This means the adopted time step size should not be larger than 0.01 , in order to get reasonably good accuracy.

In Fig. 4.2, we perform numerical simulations for initial values $\bar{\phi}_{0}=0.5$ with order parameters (4.1) and time step $\delta t=0.001$. The yellow domain, corresponding to the larger values of $\phi$, indicates the concentrated polymer segments $[16,23,36]$, and the blue region, corresponding to the smaller values of $\phi$, indicates the macromolecular microspheres (MMs). We observe the chains are partially entangled and isolated since that $\bar{\phi}_{0}=0.5$ means the polymer segments enjoy the same volume fraction as the MMs. The final equilibrium solution is obtained after $t=200$, where the polymer segments form the banded shape.

We decrease the initial values of $\bar{\phi}_{0}=0.3$ in Fig. 4.3 which means the volume fraction of the polymer segments are less than the surrounding MMs. For this case, we observe that the polymer chains are too short to entangle with each other, therefore, they graft on the surface of the MMs. The final equilibrium solution presents a very regular shape where the polymer segments accumulate together on the four corners of computed domain.

We set the intermediate value for the volume fraction, i.e., $\bar{\phi}_{0}=0.4$ in Fig. 4.4. Initially, the dynamical behaviors are very similar to the example of $\bar{\phi}_{0}=0.3$, where the polymer chains graft and are too short to entangle with each other. However, the final equilibrium shape presents a circular shape that is located inside the domain.

In Fig. 4.5, we present the evolution of the free energy functional for all three initial average of $\phi_{0}=0.3,0.4$, and 0.5 . The energy curves show the decays with time that confirms that our algorithms are unconditionally stable.

We now keep the initial value $\bar{\phi}_{0}=0.4$ but choose a different value of the order parameter $\beta=5.5$ in Fig. 4.6. In this case, the locations of the two minimizers of the bulk potential $\mathbb{F}(x)$ in (2.6) are $x_{1} \cong 0.00426$ and $x_{2} \cong 0.99571$. They are much closer to the endpoints of the domain $(0,1)$ than that of the case when $\beta=2.5\left(x_{1} \cong 0.145\right.$ and $\left.x_{2} \cong 0.855\right)$. We remark that this big $\beta$ 
can cause the code blow up very easily if the $\kappa(\phi)$ and $\mathbb{F}(\phi)$ still take the original form in (2.5) and (2.6). But in our computations, since the domain of $\mathbb{F}(\phi)$ has been extended to $(-\infty, \infty)$ in $(3.2)$, and two singularities of $\kappa(\phi)$ are avoided in (3.3), the simulations in Fig. 4.6 still present similar phase separation behaviors as Fig. 4.4 where $\beta=2.5$.

4.3. Spinodal decomposition in 3D. We continue to perform the phase separation dynamics using the second order scheme LS-BDF2 and time step $\delta t=0.001$, but in 3D space. In order to be consistent with the $2 \mathrm{D}$ case, the initial condition reads as follows,

$$
\phi(t=0)=\bar{\phi}_{0}+0.001 \operatorname{rand}(x, y, z),
$$

where the $\operatorname{rand}(x, y, z)$ is the random number in $[-1,1]$ that follows the normal distribution. We still take the default order parameters (4.1) in order to compare with the $2 \mathrm{D}$ results.

Fig. 4.7 shows the dynamical behaviors of the phase separation for the initial value $\bar{\phi}_{0}=0.5$. The dynamical evolutions are consistent with the 2D example, in which, we observe that the polymer segments are partially entangled and isolated. However, apparently, the final equilibrium solution is not a regular shape at all, far away from the banded shape in $2 \mathrm{D}$. For initial value $\bar{\phi}_{0}=0.3$, in Fig. 4.8, we observe that the polymer segments finally accumulate together to the spherical shape, as shown in $2 \mathrm{D}$ case. In Fig. 4.9 , we set the initial value $\bar{\phi}_{0}=0.4$. We observe that the polymers chains entangled with each other and finally they form the cylindrical shape.

In Fig. 4.10, we combine all of the steady solutions with $\bar{\phi}_{0}=0.3,0.4,0.5$ together and compare them with the experimental results. In order to obtain more accurate view, since the computed domain is periodic, we then plot the isosurfaces for 4 periods, i.e., $[0,8 \pi]^{3}$. It is quite clearly that all equilibrium solutions present very regular shape, i.e., the spherical shape, the cylindrical shape and the cross-pipe shape, that show qualitatively consistent features with the experimental results of sphere state, cylindrical state, and the lamellar phase, respectively (cf. $[17,36]$ ).

In Fig. 4.11, we present the evolution of the free energy functional for all three cases and observe that the energy curves decay with respect to the time.

5. Concluding Remarks. In this paper, we have developed three efficient, semi-discrete in time, first and second order linear schemes for solving the PF-HB phase field model based on a novel IEQ approach. Compared to the prevalent nonlinear schemes based on the convex splitting approaches or other nonlinear schemes, our proposed schemes conquer the inconvenience from nonlinearities by linearizing the nonlinear terms in the new way. We show that all three linear schemes developed are unconditionally energy stable. The obtained linear system is symmetric positive definite, thus one can apply any Krylov subspace methods with mass lumping as pre-conditioners for solving such systems effectively. We verify numerically that our schemes are of first and second order accuracy in time and present various $2 \mathrm{D}$ and $3 \mathrm{D}$ numerical results for some benchmark numerical simulations.

Finally, the method is general enough to be extended to develop linear schemes for a large class of gradient flow problems with complex nonlinearities in the free energy density. Although we consider only time discrete schemes in this study, the results can be carried over to any consistent finite-dimensional Galerkin approximations since the proofs are all based on a variational formulation with all test functions in the same space as the space of the trial functions.

\section{REFERENCES}

[1] K. Binder. Collective diffusion, nucleation, and spinodal decomposition in polymer mixtures. J. Chem. Phys., 79:6387, 1983.

[2] A. J. Bray. An introduction to the kinetics of first-order phase transitions. Adv. Phys., 43:357, 1994. 
[3] J. W. Cahn, C. M. Elliott, and A. Novick-Cohen. The cahn-hilliard equation with a concentration-dependent mobility: motion by minus the laplacian of the mean curvatur. European J. Appl. Math., 7:287-301, 1996.

[4] J. W. Cahn and J. E. Hilliard. Free energy of a nonuniform system. I. interfacial free energy. J. Chem. Phys., 28:258-267, 1958.

[5] R. Chen, G. Ji, X. Yang, and H. Zhang. Decoupled energy stable schemes for phase-field vesicle membrane model. J. Comput. Phys., 302:509-523, 2015.

[6] H. E. Cook. Brownian motion in spinodal decomposition mouvement brownien dans la decomposition spinodale brownsche bewegung bei der spinodalen entmischung. Acta Metall, 18:297, 1970.

[7] M. I. M. Copetti and C. M. Elliott. Numerical analysis of the cahn-hilliard equation with a logarithmic free energy. Numer. Math., 63(4):39-65, 1992.

[8] P. G. de Gennes. Scaling concepts in polymer physics. Cornell University Press, Ithaca, 1979.

[9] P. G. de Gennes. Dynamics of fluctuations and spinodal decomposition in polymer blends. J. Chem. Phys., $7: 4756,1980$

[10] A. Diegel, C. Wang, X. Wang, and S. Wise. Convergence analysis and error estimates for a second order accurate finite element method for the cahn-hilliard-navier-stokes system. Arxiv, 2016.

[11] A. Diegel, C. Wang, and S. Wise. Stability and convergence of a second order mixed finite element method for the cahn hilliard equation. IMA Journal of Numerical Analysis, 2015.

[12] C. Elliott and H. Garcke. On the cahn-hilliard equation with degenerate mobility. siam j. math. anal. SIAM J. Math. Anal., 27:404-423, 1996.

[13] D. J. Eyre. Unconditionally gradient stable time marching the Cahn-Hilliard equation. In Computational and mathematical models of microstructural evolution (San Francisco, CA, 1998), volume 529 of Mater. Res. Soc. Sympos. Proc., pages 39-46. MRS, Warrendale, PA, 1998.

[14] X. Feng and A. Prohl. Error analysis of a mixed finite element method for the cahn-hilliard equation. Numerical Mathematics, 99:47-84, 2004.

[15] X. Feng and A. Prol. Numerical analysis of the allen-cahn equation and approximation for mean curvature flows. Numer. Math., 94:33-65, 2003.

[16] M. Fialkowski and R. Holyst. Dynamics of phase separation in polymer blends revisited: morphology, spinodal, noise, and nucleation. Macromol. Theory Simul., 17:263, 2008.

[17] S. Forster, A. K. Khandpur, J. Zhao., and F. S. Bates. Complex phase behavior of polyisoprene-polystyrene diblock copolymers near the order-disorder transition. Macromolecules, 27:6922-6935, 1994.

[18] F. Guillén-González and G. Tierra. On linear schemes for a Cahn-Hilliard diffuse interface model. J. Comput. Phys., 234:140-171, 2013.

[19] D. Han, A. Brylev, X. Yang, and Z. Tan. Numerical analysis of second order, fully discrete energy stable schemes for phase field models of two phase incompressible flows. to appear, J. Sci. Comput., 2016.

[20] Y. He, Y. Liu, and T. Tang. On large time-stepping methods for the cahn-hilliard equation. Journal of Applied Numerical Mathematics, 57:616-628, 2007.

[21] J. Kim. Phase-field models for multi-component fluid flows. Comm. Comput. Phys, 12(3):613-661, 2012.

[22] J. S. Langer. An introduction to the kinetics of first-order phase transitions. Solids Far from Equilibrium, C. Godreche, Ed., Cambridge University Press, Cambridge, 18:297, 1992.

[23] X. Li, G. Ji, and H. Zhang. Phase transitions of macromolecular microsphere composite hydrogels based on the stochastic cahnhilliard equation. J. Comput. Phys., 283:81-97, 2015.

[24] C. Liu and J. Shen. A phase field model for the mixture of two incompressible fluids and its approximation by a Fourier-spectral method. Physica D, 179(3-4):211-228, 2003.

[25] J. Lowengrub and L. Truskinovsky. Quasi-incompressible cahn-hilliard fluids and topological transitions. Proc. R. Soc. Lond. A, 454:2617-2654, 1998.

[26] J. S. Lowengrub, A. Ratz, and A. Voigt. Phase field modeling of the dynamics of multicomponent vesicles spinodal decomposition coarsening budding and fission. Physical Review E, 79(3), 2009.

[27] C. Miehe, M. Hofacker, and F. Welschinger. A phase field model for rate-independent crack propagation: Robust algorithmic implementation based on operator splits. Computer Methods in Applied Mechanics and Engineering, 199:2765-2778, 2010.

[28] S. Minjeaud. An unconditionally stable uncoupled scheme for a triphasic cahn-hilliard/navier-stokes model. Commun. Comput. Phys., 29:584-618, 2013.

[29] Z. Qiao, Z. Sun, and Z. Zhang. Stability and convergence of second-order schemes for the nonlinear epitaxial growth model without slope selection. Math. Comp., 84:653674, 2015.

[30] L. Rayleigh. On the theory of surface forces-II. compressible fluids. Phil. Mag., 33:209C220, 1892.

[31] J. Shen and X. Yang. Numerical approximations of allen-cahn and cahn-hilliard equations. DCDS, Series A, 28:1169-1691, 2010.

[32] R. Spatschek, E. Brener, and A. Karma. A phase field model for rate-independent crack propagation: Robust algorithmic implementation based on operator splits. Philosophical Magazine, 91:75-95, 2010.

[33] J. van der Waals. The thermodynamic theory of capillarity under the hypothesis of a continuous density variation. 
J. Stat. Phys., 20:197-244, 1893.

[34] C. Xu and T. Tang. Stability analysis of large time-stepping methods for epitaxial growth models. Liq. Cryst., 44:1759-1779, 2006.

[35] X. Yang and D. Han. Linearly first- and second-order, unconditionally energy stable schemes for the phase field crystal equation. to appear, J. Comput. Phys., 2016.

[36] C. Yuan and H. Zhang. Self-consistent mean field model of hydrogel and its numerical simulation. Journal of Theoretical and Computational Chemistry, 12:1350048, 2013.

[37] J. Zhao, Q. Wang, and X. Yang. Numerical approximations for a phase field dendritic crystal growth model based on the invariant energy quadratization approach. to appear, Inter. J. Num. Meth. Engr., 2016.

[38] J. Zhu, L. Chen, J. Shen, and V. Tikare. Coarsening kinetics from a variable-mobility cahn-hilliard equation: Application of a semi-implicit fourier spectral method. Phys. Rev. E, 60(4):3564-3572, 1999. 